\title{
COCYCLE DEFORMATIONS AND GALOIS OBJECTS OF SEMISIMPLE HOPF ALGEBRAS OF DIMENSION 16
}

\author{
RONGCHUAN XIONG AND ZHIQIANG YU*
}

\begin{abstract}
In this article, we determine cocycle deformations and Galois objects of non-commutative and non-cocommutative semisimple Hopf algebras of dimension 16. We show that these Hopf algebras are pairwise twist inequivalent mainly by calculating their higher Frobenius-Schur indicators, and that except three Hopf algebras which are cocycle deformations of dual group algebras, none of them admit non-trivial cocycle deformations.
\end{abstract}

Keywords: Semisimple Hopf algebras; Galois objects; Cocycle deformations; Frobenius-Schur indicators.

\section{INTRODUCTION}

Let $\mathbb{k}$ be an algebraically closed field, $\mathbb{k}^{\times}:=\mathbb{k} \backslash\{0\}$, and $H$ a finite-dimensional Hopf algebra over $\mathbb{k}$. Denote by $\operatorname{Rep}(H)$ and $\operatorname{Corep}(H)$ the category of finite-dimensional $H$-modules and $H$-comudules, respectively, by $\mathbb{Z}$ the set of integer numbers, $\mathbb{Z}_{+}$the set of non-negative integers, $\mathbb{Z}_{n}:=\mathbb{Z} / n \mathbb{Z}$ for any positive integer $n$.

In 1987, Ulbrich [U1] showed that isomorphism classes of right Galois objects of $H^{*}$ are in bijective correspondence with isomorphism classes of fiber functors of the finite tensor category $\operatorname{Rep}(H)$. In practice, however, it is a challenging question to find all fiber functors. With the development of tensor category theory, especially fusion category theory [ENO1, ENO2, EGNO, O1], one can instead study rank one semisimple module categories over a fusion category $\mathcal{C}$, since semisimple module categories of rank one over $\mathcal{C}$ correspond to fiber functors on $\mathcal{C}$.

In 2003, Ostrik O1] gave an abstract and complete description of the exact indecomposable module categories $\mathcal{M}$ over a fusion category $\mathcal{C}$, that is, $\mathcal{M}$ is equivalent to the category $\mathcal{C}_{A}$ of right $A$ modules in $\mathcal{C}$ for some exact indecomposable algebra $A$ in $\mathcal{C}$. In particular, Ostrik [02 classified the exact indecomposable module categories over any group-theoretical fusion category except for an incomplete criterion of equivalence classes. Recently, the complete classification is given by Natale [Na4]. As a byproduct, fiber functors on a group-theoretical fusion category were determined in O2, Corolllary 3.4] and [Na4, Theorem 1.1].

Natale showed that semisimple Hopf algebras $H$ obtained by abelian extensions are group-theoretical, and also characterized explicitly the categories $\operatorname{Rep}(H)$ Na3. Motivated by the works mentioned above, the authors in CMNVW] determined Galois objects and cocycle deformations of non-commutative non-cocommutative semisimple Hopf algebras of dimension $p^{3}$ and $p^{2} q$ with $p, q$ distinct prime numbers, which can be obtained by abelian extensions [Ma1, Na1, Na2, ENO2]. Masuoka has already studied Galois objects and cocycle deformations of four families of semisimple Hopf algebras denoted by $\widehat{\mathcal{D}}_{4 n}, \widehat{\mathcal{T}}_{4 m}, \mathcal{A}_{4 m}$ and $\mathcal{B}_{4 m}$ in a different way, see [Ma2]. The results on Galois objects can be recovered in principle by using the techniques in [CMNVW].

In this article, we study Galois objects and cocycle deformations of non-commutative non-cocomutative semisimple Hopf algebras of dimension 16, which were classified by Kashina [Ka]. It seems possible to

2010 Mathematics Subject Classification. 16T05; 16S35; 18D10.

${ }^{*}$ Corresponding author. The authors were partially supported by the NSFC (Grant No.11771142). 
determine Galois objects of them following the method of [CMNVW], as they are obtained by abelian extensions and hence are group-theoretical. The results on Galois objects are listed as follows:

Theorem A (Theorem 3.18). Let $H$ be a semisimple Hopf algebra of dimension 16 listed in Ka, Table 1]. Then the number of Galois objects of $H^{*}$ is given as follows:

(1) $\left(H_{C: \sigma_{1}}\right)^{*} \cong H_{C: \sigma_{1}},\left(H_{c: \sigma_{1}}\right)^{*},\left(H_{B: 1}\right)^{*} \cong H_{C: 1}$ and $\left(H_{B: X}\right)^{*} \cong H_{E}$ only have one trivial Galois object.

(2) $\left(H_{E}\right)^{*} \cong H_{B: X}$ and $\left(H_{c: \sigma_{0}}\right)^{*}$ have two Galois objects.

(3) $\left(H_{C: 1}\right)^{*} \cong H_{B: 1}$ and $\left(H_{a: y}\right)^{*}$ have three Galois objects.

(4) $\left(H_{d:-1,1}\right)^{*} \cong H_{d:-1,1}$ has four Galois objects.

(5) $\left(H_{d:-1,-1}\right)^{*} \cong H_{c: \sigma_{1}}$ and $\left(H_{d: 1,-1}\right)^{*} \cong H_{b: 1}$ have five Galois objects.

(6) $\left(H_{b: x^{2} y}\right)^{*}$ and $\left(H_{b: y}\right)^{*}$ have seven Galois objects.

(7) $\left(H_{a: 1}\right)^{*}$ and $\left(H_{b: 1}\right)^{*}$ have one or two Galois objects, the number of Galois objects of $\left(H_{d: 1,1}\right)^{*} \cong$ $H_{d: 1,1}$ ranges from six to eight.

If $A$ is a right $H$-Galois object, then up to isomorphism, there exists a unique Hopf algebra $L(A, H):=\left(A^{o p} \otimes A\right)^{c o H}$ such that $A$ becomes an $(L(A, H), H)$-biGalois object [S1]. Moreover, the connection between the cocycle deformations of Hopf algebras and Galois objects has been established by Schauenburg [S1. It is of interest to determine all cocycle deformations of $H$, or equivalently to determine all Drinfeld twists of $H^{*}$.

In general, it is not easy to determine the Drinfeld twist equivalence classes of semisimple (or generally quasi-) Hopf algebras. In our cases, the Frobenius-Schur indicator is a useful tool, as it is invariant under gauge equivalences [MN, NS1]. The Frobenius-Schur indicator was first introduced by Linchenko and Montogmery [LM] for Hopf algebras, then studied extensively by Kashina, Sommerhäuer and Zhu [KSZ] for semisimple Hopf algebras, by Mason and $\mathrm{Ng}$ [MN], $\mathrm{Ng}$ and Schauenburg [NS1] for semisimple quasi-Hopf algebras, and by $\mathrm{Ng}$ and Schauenburg for spherical fusion categories [NS2]. In particular, we are able to obtain the following results mainly by computing higher Frobenius-Schur indicators.

Theorem B (Theorem 4.7). The Hopf algebras $H_{B: 1}, H_{B: X}, H_{d: 1,1}$ have only one non-trivial cocycle deformation, and other Hopf algebras listed in [Ka, Table 1] admit only trivial cocycle deformations.

It should be pointed out that the results related to $H_{B: 1}$ and $H_{C: \sigma_{1}}$ in Theorems 3.18 and 4.7 were first obtained by Masuoka [Ma2], as $\mathcal{A}_{16} \cong H_{B: 1}$ and $\mathcal{B}_{16} \cong H_{C: \sigma_{1}}$.

The paper is organized as follows. In section 2, we recall some basic notations and properties of group-theoretical fusion categories, Galois objects and cocycle deformations, abelian extensions and Frobenius-Schur indicators. In section 3, we compute all matched pairs of groups corresponding to the Hopf algebras listed in [Ka, Table 1], and then compute the number of Galois objects of them. In section 4, we mainly use the Frobenius-Schur indicators to find all the twist equivalence classes, or equivalently to determine all the cocycle deformations of them.

\section{Preliminaries}

We recall some basic knowledge of group-theoretical fusion categories, Galois objects and cocycle deformations, higher Frobenius-Schur indicators and abelian extensions of semisimple Hopf algebras.

2.1. Group-theoretical fusion categories. We follow [ENO1, EGNO, O1, O2] (also [CMNVW]) to introduce some necessary concepts and notations. Let $\mathcal{C}$ be a fusion category over $\mathbb{k}, \mathcal{M}$ be an indecomposable exact left $\mathcal{C}$-module category, $\operatorname{End}(\mathcal{M})$ be the tensor category of endofuntors of $\mathcal{M}$ and $\mathcal{C}_{\mathcal{M}}^{*}$ be the fusion category of $\mathcal{C}$-module functors of $\mathcal{M}$. The rank of $\mathcal{M}$ is the number of isomorphism classes of simple objects of $\mathcal{M}$. Observe that there is a bijective correspondence between structures of 
a $\mathcal{C}$-module category on $\mathcal{M}$ and monoidal functors $F: \mathcal{C} \rightarrow \operatorname{End}(\mathcal{M})$. Then exact $\mathcal{C}$-module categories $\mathcal{M}$ of rank one correspond to fiber functors $F: \mathcal{C} \rightarrow \operatorname{Vec} \cong \operatorname{End}(\mathcal{M})$.

A fusion category $\mathcal{C}$ is pointed if all the simple objects of $\mathcal{C}$ are invertible, then the isomorphism classes of simple objects form a finite group $G$. Therefore $\mathcal{C}$ is equivalent to $V e c_{G}^{\omega}$, the category of finite-dimensional $G$-graded vector spaces with the associativity isomorphism given by a normalized 3-cocycle $\omega \in Z^{3}\left(G, \mathbb{k}^{\times}\right)$.

Let $\mathcal{C}$ and $\mathcal{D}$ be two fusion categories. $\mathcal{C}$ and $\mathcal{D}$ are categorically Morita equivalent if there exists an exact indecomposable $\mathcal{C}$-module category $\mathcal{M}$ such that $\mathcal{D}^{\text {op }} \cong \mathcal{C}_{\mathcal{M}}^{*}$ as fusion categories.

Definition 2.1. A fusion category is said to group-theoretical if it is categorically Morita equivalent to a pointed fusion category $V e c_{G}^{\omega}$.

The exact indecomposable module categories over a group-theoretical fusion category were classified by Ostrik [02]: Every exact indecomposable module category over the category $V e c_{G}^{\omega}$ is determined by a pair $(F, \alpha)$, where $F$ is a subgroup of $G$ such that the class of $\left.\omega\right|_{F \times F \times F}$ is trivial in $H^{3}\left(F, \mathbb{k}^{\times}\right)$ and $\alpha: F \times F \rightarrow \mathbb{k}^{\times}$is a 2-cochain on $F$ satisfying $d^{2}(\alpha)=\left.\omega\right|_{F \times F \times F}$. Denote by $\mathcal{C}(G, \omega, F, \alpha)$ the corresponding group-theoretical fusion category. Every exact indecomposable module category over $\mathcal{C}(G, \omega, F, \alpha)$ is determined by a pair $(L, \beta)$, where $L$ is a subgroup of $G$ and $\beta$ is a 2-cochain on $L$ such that $d^{2}(\beta)=\left.\omega\right|_{L \times L \times L}$.

A 2-cocycle $\alpha \in Z^{2}\left(G, \mathbb{k}^{\times}\right)$is said to be non-degenerate if the twisted group algebra $\mathbb{k}_{\alpha}[G]$ is a simple algebra. The following result plays a key role in [CMNVW] to determine the Galois objects of semisimple Hopf algebras of dimension $p^{3}$ and $p^{2} q$.

Theorem 2.2. ([02, Corollary 3.4], [Na4, Theorem 1.1]) Fiber functors on $\mathcal{C}(G, \omega, F, \alpha)$ correspond to pairs $(L, \beta)$, where $L$ is a subgroup of $G$ and $\beta$ is a 2-cocycle on $L$, such that the following conditions are satisfied:

(1) The class of $\left.\omega\right|_{L \times L \times L}$ is trivial;

(2) $G=L F$;

(3) The class of the 2-cocycle $\left.\left.\alpha\right|_{F \cap L} \beta^{-1}\right|_{L \cap F}$ is non-degenerate.

Two fiber functors $(L, \beta),\left(L^{\prime}, \beta^{\prime}\right)$ are isomorphic if and only if there exists an element $g \in G$ such that $L^{\prime}=g L g^{-1}$, and the cohomology class of the 2 -cocycle $\beta^{-1} \beta^{\prime g} \Omega_{g}$ is trivial in $H^{2}\left(L, \mathbb{k}^{\times}\right)$, where $\beta^{\prime g}(h, l):=\beta^{\prime}\left(g h g^{-1}, g l g^{-1}\right)$ for $h, l \in L$ and $\Omega_{g}(a, b):=\frac{\omega\left(g a g^{-1}, g b g^{-1}, g\right) \omega(g, a, b)}{\omega\left(g a g^{-1}, g, b\right)}$ for $a, b \in G$.

Remark 2.3. From [Na4, section 3.1], for any $g \in G$, we have $d^{2}\left(\Omega_{g}\right)=\frac{\omega}{\omega^{g}}$, where $\omega^{g}(a, b, c):=$ $\omega\left(g a g^{-1}, g b g^{-1}, g c g^{-1}\right), \forall a, b, c \in G$. If the class $\left.\omega\right|_{L \times L \times L}$ is trivial and $L$ is normal, then $\left[\left.\Omega_{g}\right|_{L \times L}\right] \in$ $H^{2}\left(L, \mathbb{k}^{\times}\right)$.

\subsection{Cocycle deformations and twist deformations.}

Definition $2.4([\mathrm{EV}])$. Let $H$ be a Hopf algebra over $\mathrm{k}$. A twisting $H_{\Omega}$ of $H$ is a Hopf algebra with the same algebra structure and counit $\varepsilon$, with the coproduct and antipode given by

$$
\Delta_{\Omega}(h)=\Omega \Delta(h) \Omega^{-1}, \quad S_{\Omega}(h)=u h u^{-1}, \quad \forall h \in H,
$$

where $u \in H$ and $\Omega \in H \otimes H$ are invertible elements.

$H_{\Omega}$ is well-defined if and only if $\Omega \in H \otimes H$ is a 2-pseudo-cocycle, that is, $\partial_{2}(\Omega)$ lies in the centralizer of $(\Delta \otimes$ id $) \Delta(H)$ in $H \otimes H \otimes H$, where

$$
\partial_{2}(\Omega):=(i d \otimes \Delta)\left(\Omega^{-1}\right)\left(1 \otimes \Omega^{-1}\right)(\Omega \otimes 1)(\Delta \otimes i d)(\Omega) .
$$

$\Omega$ is a 2 -cocycle if and only if $\partial_{2}(\Omega)=1 \otimes 1 \otimes 1,(\varepsilon \otimes i d) \Omega=(i d \otimes \varepsilon) \Omega=1$. In this case, we call $\Omega$ a Drinfeld twist of $H$ denoted by $J$ and $H^{J}:=H_{\Omega}$ is a (Drinfeld) twist deformation of $H$. 
Let $H, H^{\prime}$ be two Hopf algebras. Then $H$ and $H^{\prime}$ are said to be twist equivalent, if $H^{\prime} \cong H^{J}$ for some Drinfeld twist $J$ of $H$. Furthermore, if $H$ and $H^{\prime}$ are finite-dimensional, then they are twist equivalent if and only if $\operatorname{Rep}(H) \cong \operatorname{Rep}\left(H^{\prime}\right)$ as tensor categories [S1].

The properties like the semisimplicity or Grothendieck rings of Hopf algebras are preserved under twisting deformations. Moreover,

Theorem 2.5. ([Ni, Theorem 4.1]) Let $H$ and $L$ be Hopf algebras over $\mathbb{k}$. Denote by $G r(H)$ and $\operatorname{Gr}(L)$ the Grothendieck rings of $H$ and $L$, respectively. Then $\operatorname{Gr}(H) \cong G r(L)$ as fusion rings if and only if $H$ is a twisting of $L$.

Now we introduce the dual concept of the Drinfeld twist. Let $H$ be a Hopf algebra and $\sigma: H \otimes H \rightarrow \mathbb{k}$ be a map. Then $\sigma$ is said to be a Hopf 2-cocycle of $H$, if it is a convolution invertible morphism satisfying

$$
\sigma(x, 1)=\varepsilon(x)=\sigma(1, x), \quad \sigma\left(x_{(1)}, y_{(1)}\right) \sigma\left(x_{(2)} y_{(2)}, z\right)=\sigma\left(y_{(1)}, z_{(1)}\right) \sigma\left(x, y_{(2)} z_{(2)}\right), \quad \forall x, y, z \in H .
$$

Given a 2-cocycle $\sigma: H \otimes H \rightarrow \mathbb{k}$. Denote by $\sigma^{-1}$ the convolution inverse of $\sigma$. We can construct a new Hopf algebra $\left(H^{\sigma}, m^{\sigma}, 1, \Delta, \epsilon, S^{\sigma}\right)$, where $H^{\sigma}=H$ as coalgebras, and

$$
\begin{gathered}
m^{\sigma}(x \otimes y)=\sigma\left(x_{(1)}, y_{(1)}\right) x_{(2)} y_{(2)} \sigma^{-1}\left(x_{(3)}, y_{(3)}\right), \\
S^{\sigma}(x)=\sum \sigma\left(x_{(1)}, S\left(x_{(2)}\right)\right) S\left(x_{(3)}\right) \sigma^{-1}\left(S\left(x_{(4)}\right), x_{(5)}\right), \quad \forall x, y \in H .
\end{gathered}
$$

Definition 2.6. We call $H^{\sigma}$ the cocycle deformation of $H$ by $\sigma$.

A 2-cocycle deformation $H^{\sigma}$ of $H$ by $\sigma$ is said to be trivial if $H^{\sigma} \cong H$ as Hopf algebras.

Remark 2.7 ([S1]). Given a Hopf 2-cocycle $\sigma: H \otimes H \rightarrow \mathbb{k}$, there exists an algebra $H_{\sigma}$ with the multiplication given by:

$$
x \cdot{ }_{\sigma} y=\sigma\left(x_{(1)}, y_{(1)}\right) x_{(2)} y_{(2)}, \quad x, y \in H .
$$

Moreover, $\Delta: H_{\sigma} \rightarrow H_{\sigma} \otimes H$ is an algebra map and then $H_{\sigma}$ becomes a right $H$-comodule algebra and $\left(H_{\sigma}\right)^{\text {co } H} \cong \mathbb{k}$.

\subsection{Hopf Galois objects.}

Definition 2.8. Let $H, L$ be Hopf algebras over $\mathbb{k}$. A right $H$-Hopf comodule algebra $A$ is a right $H$-Galois object if $A^{c o H}=\mathbb{k}$ and the Hopf-Galois map $\beta: A \otimes A \rightarrow A \otimes H, \beta(a \otimes b)=a b_{(0)} \otimes b_{(1)}$, is bijective. A left $L$-Galois object is defined analogously. An $(L, H)$-biGalois object $A$ is a right $H$ Galois object and a left $L$-Galois object, and the left $L$-comodule and right $H$-comodule morphisms are compatible.

Given a right $H$-Galois object $A$, there is a Hopf algebra $L:=L(A, H)$ attached to the pair $(A, H)$ such that $A$ becomes an $(L, H)$-biGalois object [S1]. The Hopf algebra $L(A, H)$ is unique up to isomorphism. A right $H$-Galois object $A$ is trivial if $A \cong H$ as right $H$-comodule algebras. In this special case, $L \cong H$ as Hopf algebras.

Remark 2.9. If $\sigma: H \otimes H \rightarrow \mathbb{k}$ is a Hopf 2-cocycle, then the algebra $H_{\sigma}$ is an $\left(H^{\sigma}, H\right)$-biGalois object.

Let $H$ and $L$ be finite-dimensional Hopf algebras over $\mathbb{k}$. Then $H$ and $L$ are Morita-Takeuchi equivalent, if $\operatorname{Corep}(H) \cong \operatorname{Corep}(L)$ as finite tensor categories.

Theorem 2.10. ([S1, Corollary 5.7]) Let $H, L$ be finite-dimensional Hopf algebras. Then $H$ and $L$ are Morita-Takeuchi equivalent, if and only if $H$ is a cocycle deformation of $L$, if and only if there exists a $(H, L)$-biGalois object $A$ such that $L \cong L(A, H)$. 
Proposition 2.11. ([U1, Theorem 1.7]) Let $H$ be a finite-dimensional Hopf algebra over $\mathbb{k}$. Then there is a bijective correspondence between the following sets:

(1) The set of right Galois objects of $H^{*}$;

(2) The set of fiber functors on $\operatorname{Rep}(H)$.

\subsection{Higher Frobenius-Schur indicators.}

Definition 2.12 ([LM, $\mathrm{KSZ}]$ ). Let $H$ be a semisimple Hopf algebra, $\Lambda \in H$ be an integral such that $\varepsilon(\Lambda)=1, V$ be a finite-dimensional representation of $H$, and $\chi$ be its character. For any non-negative integer $n$, the $n$-th Frobenius-Schur indicator $\nu_{n}(\chi)$ of $V$ is given by

$$
\nu_{n}(\chi):=\chi\left(\Lambda^{[n]}\right),
$$

where $\Lambda^{[n]}:=\Lambda_{(1)} \cdots \Lambda_{(n)}$ (here $\Lambda^{[0]}:=1$ ) is the $n$-th Sweedler power of $\Lambda$.

Remark 2.13. The Frobenius-Schur indicator is also well-defined for semisimple quasi-Hopf algebras and spherical fusion categories, see [NS1, NS2] for details.

Remark 2.14. Let $H$ be a Hopf algebra fitting into an abelian extension : $\mathbb{k} \rightarrow \mathbb{k}^{\Gamma} \rightarrow H \rightarrow \mathbb{k}[F] \rightarrow \mathbb{k}$. Then the integral element $\Lambda$ of $H$ satisfying $\varepsilon(\Lambda)=1$ is given by

$$
\Lambda=\frac{1}{|F|} \Sigma_{g \in F} \delta_{1} \# g
$$

where $\delta_{1}$ is the orthogonal primitive idempotent satisfying $\left\langle\delta_{1}, g\right\rangle=\delta_{1, g}$, for any $g \in \Gamma$.

Theorem 2.15. ([MN, Theorem 4.1], [NS1, Theorem 4.1]) The Frobenius-Schur indicators are invariant under gauge transformations of semisimple (quasi)-Hopf algebras.

The Frobenius-Schur indicator is a gauge invariant, so it is useful to distinguish whether two semisimple Hopf algebras are twist inequivalent.

2.5. Abelian extensions of Hopf algebras. We follow [H, $\mathrm{K}$, Ma3, Na3] to introduce some necessary notations and concepts on abelian extensions (see also [CMNVW, section 3]). Let $F, \Gamma$ be finite groups. A matched pair $(F, \Gamma, \triangleleft, \triangleright)$ consists of two actions satisfying the following compatible conditions: $\forall s, t \in \Gamma, \forall x, y \in F$,

$$
s \triangleright(x y)=(s \triangleright x)((s \triangleleft x) \triangleright y), \quad(s t) \triangleleft x=(s \triangleleft(t \triangleright x))(t \triangleleft x) .
$$

Let $(F, \Gamma, \triangleleft, \triangleright)$ be a matched pair of groups. The bicrossed product $F \bowtie \Gamma$ of $F$ and $\Gamma$ is a group with unit $1 \bowtie 1$ and multiplication given by

$$
(x \bowtie s)(y \bowtie t)=x(s \triangleright y) \bowtie(s \triangleleft y) t, \quad \forall x, y \in F, s, t \in \Gamma .
$$

Obviously, $F \times 1$ and $1 \times \Gamma$ are subgroups of $F \bowtie \Gamma$ and isomorphic to $F$ and $\Gamma$, respectively. If we do not distinguish the subgroups $F \times 1,1 \times \Gamma$ from $F, \Gamma$, then $F \bowtie \Gamma$ admits an exact factorization $F \bowtie \Gamma=F \Gamma$.

Conversely, if a finite group $G$ admits an exact factorization $G=F^{\prime} \Gamma^{\prime}$, where $F^{\prime} \cong F$ and $\Gamma^{\prime} \cong \Gamma$ as groups, then any $g \in G$ can be written uniquely as $x s$, where $x \in F, s \in \Gamma$. Henceforth, the group multiplication implies $(x s)(y t)=\left(x y^{\prime}\right)\left(s^{\prime} t\right)$, for unique $y^{\prime} \in F, s^{\prime} \in \Gamma$, which gives two actions $\triangleleft: \Gamma \times F \rightarrow \Gamma,(s, y) \mapsto s^{\prime}$, and $\triangleright: \Gamma \times F \rightarrow F(s, y) \mapsto y^{\prime}$, such that $(F, \Gamma, \triangleleft, \triangleright)$ is a matched pair. It is easy to see that these actions are determined by the relations

$$
s y=y^{\prime} s^{\prime}=(s \triangleright y)(s \triangleleft y), \quad y \in F, s \in \Gamma .
$$

Let $(F, \Gamma, \triangleleft, \triangleright)$ be a matched pair of groups, $\left\{e_{s}\right\}_{s \in \Gamma}$ be the dual primitive orthogonal basis in $\mathbb{k}^{\Gamma}$. The action $\triangleleft: \Gamma \times F \rightarrow \Gamma$ corresponds to an action $\rightarrow: F \times \mathbb{k}^{\Gamma} \rightarrow \mathbb{k}^{\Gamma}$ so that

$$
a \rightarrow e_{x}=e_{x \triangleleft a^{-1}}, \quad a \in F, x \in \Gamma .
$$


Let $\sigma: F \times F \rightarrow\left(\mathbb{k}^{\Gamma}\right)^{\times}$be a normalized 2-cocycle of the group $F$ with coeffients in $\left(\mathbb{k}^{\Gamma}\right)^{\times}$. We write $\sigma:=\sum_{g \in \Gamma} \sigma_{g} e_{g}$. Then

$$
\sigma_{s \triangleleft x}(y, z) \sigma_{s}(x, y z)=\sigma_{s}(x y, z) \sigma_{s}(x, y), \quad \sigma_{1}(x, y)=\sigma_{s}(x, 1)=\sigma_{s}(1, y)=1, \quad \forall x, y, z \in F .
$$

Dually, let $\left\{\delta_{x}\right\}_{x \in F}$ be the dual primitive orthogonal basis in $\mathbb{k}^{F}$; the action $\triangleright: \Gamma \times F \rightarrow F$ corresponds to an action $\leftarrow: \mathbb{k}^{F} \times \Gamma \rightarrow \mathbb{k}^{F}$ so that

$$
\delta_{x}<a=\delta_{a^{-1} \triangleright x}, \quad a \in \Gamma, x \in F .
$$

Let $\tau=\sum_{x \in F} \tau_{x} \delta_{x}: \Gamma \times \Gamma \rightarrow\left(\mathbb{k}^{F}\right)^{\times}$be a normalized 2-cocycle of $\Gamma$ with coefficients in $\left(\mathbb{k}^{F}\right)^{\times}$. We write $\tau:=\sum_{g \in F} \tau_{g} e_{g}$. Then

$$
\tau_{x}(s t, u) \tau_{u \triangleright x}(s, t)=\tau_{x}(s, t u) \tau_{x}(t, u), \quad \tau_{1}(s, t)=\tau_{x}(s, 1)=\tau_{x}(1, t)=1, \quad \forall s, t, u \in \Gamma .
$$

Let $\mathbb{k}^{\Gamma \tau} \#_{\sigma} \mathbb{k}[F]$ denote the vector space $\mathbb{k}^{\Gamma} \otimes \mathbb{k}[F]$ with multiplication and comultiplication given by

$$
\begin{gathered}
\left(e_{g} \# x\right)\left(e_{h} \# y\right)=\delta_{g \triangleleft x, h} \sigma_{g}(x, y) e_{g} \# x y, \\
\Delta\left(e_{g} \# x\right)=\sum_{s t=g} \tau_{x}(s, t) e_{s} \#(t \triangleright x) \otimes e_{t} \# x, \quad g, h \in \Gamma, x, y \in F .
\end{gathered}
$$

If $\sigma$ and $\tau$ obey some certain compatible conditions, then $H:=\mathbb{k}^{\Gamma \tau} \#_{\sigma} \mathbb{k}[F]$ is a semisimple Hopf algebra fitting into an exact sequence of Hopf algebras $\mathbb{k} \rightarrow \mathbb{k}^{\Gamma} \rightarrow H \rightarrow \mathbb{k}[F] \rightarrow \mathbb{k}$. We say $H$ is an abelian extension associated to the matched pair $(F, \Gamma, \triangleleft, \triangleright)$. Moreover, every Hopf algebra fitting into such an exact sequence can be described in this way. See e.g. [Ma3, Part 1] for details.

Given a matched pair $(F, \Gamma, \triangleleft, \triangleright)$, denote by $\operatorname{Opext}\left(\mathbb{k}^{\Gamma}, \mathbb{k}[F]\right)$ the set of equivalence classes of abelian extensions $\mathbb{k}_{\mathrm{k}} \rightarrow \mathbb{k}^{\Gamma} \rightarrow \mathbb{k}^{\Gamma \tau} \#_{\sigma} \mathbb{k}_{k}[F] \rightarrow \mathbb{k}[F] \rightarrow \mathbb{k}$, which is a finite group under the Baer product of extensions. By $[\underline{\mathrm{K}},(3.14)]$, there is an exact sequence

$$
\begin{aligned}
& 0 \rightarrow H^{1}\left(F \bowtie \Gamma, \mathbb{k}^{\times}\right) \stackrel{\text { res }}{\longrightarrow} H^{1}\left(F, \mathbb{k}^{\times}\right) \oplus H^{1}\left(\Gamma, \mathbb{k}^{\times}\right) \rightarrow \operatorname{Aut}\left(\mathbb{k}^{\Gamma \tau} \#_{\sigma} \mathbb{k}[F]\right) \\
& \rightarrow H^{2}\left(F \bowtie \Gamma, \mathbb{k}^{\times}\right) \stackrel{\text { res }}{\longrightarrow} H^{2}\left(F, \mathbb{k}^{\times}\right) \oplus H^{2}\left(\Gamma, \mathbb{k}^{\times}\right) \rightarrow \operatorname{Opext}\left(\mathbb{k}^{\Gamma}, \mathbb{k}_{k}[F]\right) \\
& \stackrel{\bar{\omega}^{\rightarrow}}{\rightarrow} H^{3}\left(F \bowtie \Gamma, \mathbb{k}^{\times}\right) \stackrel{\text { res }}{\longrightarrow} H^{3}\left(F, \mathbb{k}^{\times}\right) \oplus H^{3}\left(\Gamma, \mathbb{k}^{\times}\right) \rightarrow \cdots
\end{aligned}
$$

In particular, the element $[\tau, \sigma] \in \operatorname{Opext}\left(\mathbb{k}^{\Gamma}, \mathbb{k}[F]\right)$ is mapped under $\bar{\omega}$ onto a 3 -cocycle $\omega(\tau, \sigma) \in$ $Z^{3}\left(F \bowtie \Gamma, \mathbb{k}^{\times}\right)$, which is defined by:

$$
\omega(\tau, \sigma)(a, b, c)=\tau_{\pi(c)}(p(a) \triangleleft \pi(b), p(b)) \sigma_{p(a)}(\pi(b), p(b) \triangleright \pi(c)), \quad \forall a, b, c \in F \bowtie \Gamma,
$$

where $\pi: F \bowtie \Gamma \rightarrow F, g \bowtie h \mapsto g$ and $p: F \bowtie \Gamma \rightarrow \Gamma, g \bowtie h \mapsto h$ are projections.

Theorem 2.16. ([Na3, Theorem 1.3]) Let $(F, \Gamma, \triangleleft, \triangleright)$ be a matched pair of groups and $H$ be a Hopf algebra fitting into an abelian extension $\mathbb{k} \rightarrow \mathbb{k}^{\Gamma} \rightarrow H \rightarrow \mathbb{k}[F] \rightarrow \mathbb{k}$ associated to $(F, \Gamma, \triangleleft, \triangleright)$. Then $H$ is group-theoretical and $\operatorname{Rep}(H) \cong \mathcal{C}(F \bowtie \Gamma, \omega(\tau, \sigma), F, 1)$ as fusion categories.

\section{Galois objects of Semisimple Hopf algebras of dimension 16}

We study Galois objects of non-commutative and non-cocommutative semisimple Hopf algebras of dimension 16 listed in [Ka, Table 1]. 
3.1. Singer pairs and matched pairs. Let $H$ be a semisimple Hopf algebra of dimension 16 in $[\mathrm{Ka}$, Table 1]. Kashina [Ka] showed that $H$ fits into a cocentral abelian exact sequence of Hopf algebras

$$
\mathbb{k}^{\Gamma} \hookrightarrow H \rightarrow \mathbb{k}[F]
$$

where $F:=\langle t\rangle \cong \mathbb{Z}_{2}$ and $\Gamma$ is a group of order 8 .

Moreover, $H$ is a bicrossed product $\mathbb{k}^{\Gamma \rho, \theta} \# \rightarrow, \sigma k[F]$ with a non-trivial action $\rightarrow: \mathbb{k}[F] \otimes \mathbb{k}^{\Gamma} \rightarrow \mathbb{k}^{\Gamma}$ and a trivial coaction $\rho: \mathbb{k}[F] \rightarrow \mathbb{k}[F] \otimes \mathbb{k}^{\Gamma}$, a Singer 2-cocycle $\sigma: \mathbb{k}[F] \otimes \mathbb{k}[F] \rightarrow \mathbb{k}^{\Gamma}$ and a dual Singer 2-cocycle $\theta: \mathbb{k}[F] \rightarrow \mathbb{k}^{\Gamma} \otimes \mathbb{k}^{\Gamma}$. The pair $\left(\mathbb{k}[F], \mathbb{k}^{\Gamma}, \rightarrow, \rho\right)$ is called a Singer pair.

There is a bijective correspondence between the Singer pair $\left(\mathbb{k}[F], \mathbb{k}^{\Gamma}, \rightarrow, \rho\right)$ and the matched pair $(F, \Gamma, \triangleleft, \triangleright)$. In our cases, the trivial coaction $\rho: \mathbb{k}[F] \rightarrow \mathbb{k}[F] \otimes \mathbb{k}^{\Gamma}$ implies that $\triangleright: \Gamma \otimes F \rightarrow \Gamma$ is trivial and the module action $\rightarrow: F \otimes \mathbb{k}^{\Gamma} \rightarrow \mathbb{k}^{\Gamma}$ corresponds to the action $\triangleleft: \Gamma \otimes F \rightarrow F$ so that

$$
x \rightarrow \delta_{s}=\delta_{s \triangleleft x^{-1}}, \quad \forall x \in F, s \in \Gamma .
$$

Given a Singer 2-cocycle $\sigma: \mathbb{k}[F] \otimes \mathbb{k}[F] \rightarrow \mathbb{k}^{\Gamma}$ and a dual Singer 2-cocycle $\theta: \mathbb{k}[F] \rightarrow \mathbb{k}^{\Gamma} \otimes \mathbb{k}^{\Gamma}$, the corresponding element $[\tau, \sigma] \in \operatorname{Opext}\left(\mathbb{k}^{\Gamma}, \mathbb{k}[F]\right)$ is given by

$$
\begin{aligned}
& \tau_{f}(g, h)=\theta(f)(g, h), \quad \forall g, h \in \Gamma, f \in F, \\
& \sigma_{s}(x, y)=\sigma(x, y)(s), \quad \forall s \in \Gamma, x, y \in F .
\end{aligned}
$$

Let $H$ be a Hopf algebra listed in [Ka, Table 1]. Then $H \cong \mathbb{k}^{\Gamma \rho, \theta} \# \rightarrow, \sigma \mathbb{k}[F]$ for some Singer pair $\left(\mathbb{k}[F], \mathbb{k}^{\Gamma}, \rightarrow, \rho\right)$, Singer 2-cocycle $\sigma$ and dual Singer 2-cocycle $\theta$. We shall describe the bicrossed product $F \bowtie \Gamma$ and the 3-cocycle $\omega(\sigma, \tau) \in Z^{3}\left(F \bowtie \Gamma, \mathbb{k}^{\times}\right)$. We proceed this case by case following the classification of Kashina.

Case I: $\Gamma=\mathbb{Z}_{4} \times \mathbb{Z}_{2}=\langle x\rangle \times\langle y\rangle$. Let $\left\{e_{p, q}\right\}_{0 \leq p \leq 3,0 \leq q \leq 1}$ be the dual basis in $\mathbb{k}^{\Gamma}$, that is, $\left\langle e_{p, q}, x^{i} y^{j}\right\rangle=$ $\delta_{p, i} \delta_{q, j}$ for $0 \leq i<4,0 \leq j<2$. In this case, $\theta(t)=\sum_{i j p q}(-1)^{j p} e_{i j} \otimes e_{p q}$. Then

$$
\tau_{t^{s}}\left(x^{i} y^{j}, x^{k} y^{l}\right)=(-1)^{j k s} .
$$

Indeed, $\tau\left(x^{r} y^{s}, x^{k} y^{l}\right)(t)=\theta(t)\left(x^{r} y^{s}, x^{k} y^{l}\right)=\sum_{i j p q}(-1)^{j p}\left\langle e_{i, j} \otimes e_{p, q}, x^{r} y^{s} \otimes x^{k} y^{l}\right\rangle=(-1)^{s k}$.

Case (a): The action is given by $t \rightarrow e_{i, j}=e_{i+2 j, j}$ and $\sigma(t, t)=\sum_{p, q}(-1)^{k p+l q} e_{p, q}$ for $k, l=0,1$. In this case, the Singer pair forms the Hopf algebra $H_{k, l}$.

We claim that $F \bowtie \Gamma$ is isomorphic to

$$
D_{8} * \mathbb{Z}_{4}=G_{7}:=\left\langle x, y, t \mid x^{4}=y^{2}=t^{2}=1,[x, y]=[x, t]=1,[y, t]=x^{2}\right\rangle .
$$

Indeed, $x \triangleright t=y \triangleright t=t$, and $x \triangleleft t=x, y \triangleleft t=x^{2} y$ since the action $\triangleright$ is trivial and

$$
\begin{aligned}
\left\langle x \triangleleft t, e_{i, j}\right\rangle=\left\langle x, t \rightarrow e_{i, j}\right\rangle & =\left\langle x, e_{i+2 j, j}\right\rangle=\delta_{1, i+2 j} \delta_{0, j}, \\
\left\langle y \triangleleft t, e_{i j}\right\rangle=\left\langle y, t \rightarrow e_{i j}\right\rangle & =\left\langle y, e_{i+2 j, j}\right\rangle=\delta_{0, i+2 j} \delta_{1, j} .
\end{aligned}
$$

Then the claim follows by the following relations

$$
(1 \bowtie x)(t \bowtie 1)=t \bowtie x, \quad(1 \bowtie y)(t \bowtie 1)=(y \triangleright t) \bowtie(y \triangleleft t)=t \bowtie x^{2} y .
$$

Now we calculate $\omega(\sigma, \tau) \in Z^{3}\left(F \bowtie \Gamma, \mathbb{k}^{\times}\right)$.

(1) $H_{a: 1}:=H_{0,0}$. Then $\sigma$ is trivial, that is, $\sigma_{x^{i} y^{j}}\left(t^{r}, t^{s}\right)=1$. Let $a=t^{q} x^{i} y^{j}, b=t^{r} x^{k} y^{l}$ and $c=t^{s} x^{m} y^{n}$ for some $q, r, s, j, l, n \in \mathbb{Z}_{2}, i, k, m \in \mathbb{Z}_{4}$. By the formula (1), we have

$$
\begin{aligned}
& \omega(\tau, \sigma)(a, b, c)=\tau_{\pi(c)}(p(a) \triangleleft \pi(b), p(b)) \sigma_{p(a)}(\pi(b), p(b) \triangleright \pi(c)) \\
& =\tau_{t^{s}}\left(\left(x^{i} \triangleleft t^{r}\right)\left(y^{j} \triangleleft t^{r}\right), x^{k} y^{l}\right)=\tau_{t^{s}}\left(x^{i+2 r j} y^{j}, x^{k} y^{l}\right)=(-1)^{j k s} .
\end{aligned}
$$


(2) $H_{a: y}:=H_{0,1}$. Since $\sigma(t, t)=\Sigma_{p, q}(-1)^{q} e_{p, q}$, it follows that $\sigma_{x^{i} y^{j}}\left(t^{r}, t^{s}\right)=(-1)^{j r s}$. Then

$$
\begin{aligned}
& \omega(\tau, \sigma)(a, b, c)=\tau_{\pi(c)}(p(a) \triangleleft \pi(b), p(b)) \sigma_{p(a)}(\pi(b), p(b) \triangleright \pi(c)) \\
& =\tau_{t^{s}}\left(\left(x^{i} \triangleleft t^{r}\right)\left(y^{j} \triangleleft t^{r}\right), x^{k} y^{l}\right) \sigma_{x^{i} y^{j}}\left(t^{r}, t^{s}\right)=\tau_{t^{s}}\left(x^{i+2 r j} y^{j}, x^{k} y^{l}\right)(-1)^{j r s} \\
& =(-1)^{j k s}(-1)^{j r s}=(-1)^{j(k+r) s} .
\end{aligned}
$$

Case (b): The action is given by $t \rightarrow e_{i j}=e_{-i, j}$ and $\sigma(t, t)=\sum_{p, q}(-1)^{k p+l q} e_{p, q}$ for $0 \leq k, l \leq 1$. In this case, the Singer pair forms the Hopf algebra $H_{k, l}$.

We claim that $F \bowtie \Gamma$ is isomorphic to

$$
D_{8} \times \mathbb{Z}_{2}=G_{8}:=\left\langle x, t, y \mid x^{4}=t^{2}=y^{2}=1, t x=x^{-1} t,[x, y]=[t, y]=1\right\rangle .
$$

Indeed, $x \triangleright t=y \triangleright t=t, x \triangleleft t=x^{-1}$ and $y \triangleleft t=y$ since the action $\triangleright$ is trivial and

$$
\begin{aligned}
& \left\langle x \triangleleft t, e_{i j}\right\rangle=\left\langle x, t \rightarrow e_{i j}\right\rangle=\left\langle x, e_{-i, j}\right\rangle=\delta_{1,-i} \delta_{0, j}, \\
& \left\langle y \triangleleft t, e_{i j}\right\rangle=\left\langle y, t \rightarrow e_{i j}\right\rangle=\left\langle y, e_{-i, j}\right\rangle=\delta_{0,-i} \delta_{1, j} .
\end{aligned}
$$

Then the claim follows by the following relations

$$
(1 \bowtie x)(t \bowtie 1)=(x \triangleright t) \bowtie(x \triangleleft t)=t \bowtie x^{-1}, \quad(1 \bowtie y)(t \bowtie 1)=t \bowtie y .
$$

Now we calculate $\omega(\sigma, \tau) \in Z^{3}\left(F \bowtie \Gamma, \mathbb{k}^{\times}\right)$.

(1) $H_{b: 1}:=H_{0,0} \cong H_{1,0}$. Then $\sigma$ is trivial. Let $a=t^{q} x^{i} y^{j}, b=t^{r} x^{k} y^{l}$ and $c=t^{s} x^{m} y^{n}$. Then

$$
\begin{aligned}
& \omega(\tau, \sigma)(a, b, c)=\tau_{\pi(c)}(p(a) \triangleleft \pi(b), p(b)) \sigma_{p(a)}(\pi(b), p(b) \triangleright \pi(c)) \\
& =\tau_{t^{s}}\left(\left(x^{i} \triangleleft t^{r}\right)\left(y^{j} \triangleleft t^{r}\right), x^{k} y^{l}\right)=\tau_{t^{s}}\left(x^{(-1)^{r} i} y^{j}, x^{k} y^{l}\right)=(-1)^{j k s} .
\end{aligned}
$$

(2) $H_{b: y}:=H_{0,1}$. Since $\sigma(t, t)=\sum_{p, q}(-1)^{q} e_{p, q}$, it follows that $\sigma_{x^{i} y^{j}}\left(t^{r}, t^{s}\right)=(-1)^{j r s}$. Then

$$
\begin{aligned}
& \omega(\tau, \sigma)(a, b, c)=\tau_{\pi(c)}(p(a) \triangleleft \pi(b), p(b)) \sigma_{p(a)}(\pi(b), p(b) \triangleright \pi(c)) \\
& =\tau_{t^{s}}\left(\left(x^{i} \triangleleft t^{r}\right)\left(y^{j} \triangleleft t^{r}\right), x^{k} y^{l}\right) \sigma_{x^{i} y^{j}}\left(t^{r}, t^{s}\right)=\tau_{t^{s}}\left(x^{(-1)^{r} i} y^{j}, x^{k} y^{l}\right)(-1)^{j r s} \\
& =(-1)^{j k s}(-1)^{j r s}=(-1)^{j(k+r) s} .
\end{aligned}
$$

(3) $H_{b: x^{2} y}:=H_{1,1}$. Since $\sigma(t, t)=\sum_{p, q}(-1)^{p+q} e_{p, q}$, it follows $\sigma_{x^{i} y^{j}}\left(t^{r}, t^{s}\right)=(-1)^{(i+j) r s}$. Then

$$
\begin{aligned}
& \omega(\tau, \sigma)(a, b, c)=\tau_{\pi(c)}(p(a) \triangleleft \pi(b), p(b)) \sigma_{p(a)}(\pi(b), p(b) \triangleright \pi(c)) \\
& =\tau_{t^{s}}\left(\left(x^{i} \triangleleft t^{r}\right)\left(y^{j} \triangleleft t^{r}\right), x^{k} y^{l}\right) \sigma_{x^{i} y^{j}}\left(t^{r}, t^{s}\right)=\tau_{t^{s}}\left(x^{(-1)^{r} i} y^{j}, x^{k} y^{l}\right)(-1)^{(i+j) r s} \\
& =(-1)^{j k s}(-1)^{(i+j) r s}=(-1)^{(j k+j r+i r) s} .
\end{aligned}
$$

Case (c): The action is given by $t \rightarrow e_{i, j}=e_{i, i+j}$ and $\sigma(t, t)=\sum_{p, q}(-1)^{\frac{p(p-1)}{2}} \theta^{k p} e_{p, q}$ for $\theta$ a primitive 4-th root of unity. The Singer pair forms the Hopf algebra $H_{k}$ for $0 \leq k \leq 1$.

We claim that $F \bowtie \Gamma$ is isomorphic to

$$
G_{6}:=\left\langle x, t, y \mid x^{4}=t^{2}=y^{2}=1,[y, x]=[y, t]=1,[x, t]=y\right\rangle .
$$

Indeed, $x \triangleright t=y \triangleright t=t, x \triangleleft t=x y$ and $y \triangleleft t=y$, since the action $\triangleright$ is trivial and

$$
\begin{aligned}
\left\langle x \triangleleft t, e_{i, j}\right\rangle=\left\langle x, t \rightarrow e_{i, j}\right\rangle & =\left\langle x, e_{i, i+j}\right\rangle=\delta_{1, i} \delta_{0, i+j}, \\
\left\langle y \triangleleft t, e_{i, j}\right\rangle=\left\langle y, t \rightarrow e_{i j}\right\rangle & =\left\langle y, e_{i, i+j}\right\rangle=\delta_{0, i} \delta_{1, i+j} .
\end{aligned}
$$

Then the claim follows by the following relations

$$
(1 \bowtie x)(t \bowtie 1)=(x \triangleright t) \bowtie(x \triangleleft t)=t \bowtie x y, \quad(1 \bowtie y)(t \bowtie 1)=t \bowtie y .
$$

Now we calculate $\omega(\sigma, \tau) \in H^{3}\left(F \bowtie \Gamma, \mathbb{k}^{\times}\right)$. 
(1) $H_{c: \sigma_{0}}:=H_{0}$. Since $\sigma(t, t)=\sum_{p, q}(-1)^{\frac{p(p-1)}{2}} e_{p, q}$, it follows that $\sigma_{x^{i} y^{j}}\left(t^{r}, t^{s}\right)=(-1)^{\frac{i(i-1)}{2}} r s$. Then

$$
\begin{aligned}
& \omega(\tau, \sigma)(a, b, c)=\tau_{\pi(c)}(p(a) \triangleleft \pi(b), p(b)) \sigma_{p(a)}(\pi(b), p(b) \triangleright \pi(c)) \\
& =\tau_{t^{s}}\left(\left(x^{i} \triangleleft t^{r}\right)\left(y^{j} \triangleleft t^{r}\right), x^{k} y^{l}\right) \sigma_{x^{i} y^{j}}\left(t^{r}, t^{s}\right) \\
& =\tau_{t^{s}}\left(x^{i} y^{r i+j}, x^{k} y^{l}\right)(-1)^{\frac{i(i-1)}{2} r s} \\
& =(-1)^{(r i+j) k s}(-1)^{\frac{i(i-1)}{2} r s}=(-1)^{\frac{2(r i+j) k s+i(i-1) r s}{2}} .
\end{aligned}
$$

(2) $H_{c: \sigma_{1}}:=H_{1}$. Since $\sigma(t, t)=\sum_{p, q}(-1)^{\frac{p(p-1)}{2}} \theta^{p} e_{p, q}$, it follows that $\sigma_{x^{i} y^{j}}\left(t^{r}, t^{s}\right)=(-1)^{\frac{i(i-1)}{2}} r s \theta^{i r s}$. Then

$$
\begin{aligned}
& \omega(\tau, \sigma)(a, b, c)=\tau_{\pi(c)}(p(a) \triangleleft \pi(b), p(b)) \sigma_{p(a)}(\pi(b), p(b) \triangleright \pi(c)) \\
& =\tau_{t^{s}}\left(\left(x^{i} \triangleleft t^{r}\right)\left(y^{j} \triangleleft t^{r}\right), x^{k} y^{l}\right) \sigma_{x^{i} y^{j}}\left(t^{r}, t^{s}\right) \\
& =\tau_{t^{s}}\left(x^{i} y^{r i+j}, x^{k} y^{l}\right)(-1)^{\frac{i(i-1)}{2} r s} \theta^{i r s} \\
& =(-1)^{(r i+j) k s}(-1)^{\frac{i(i-1)}{2} r s} \theta^{i r s}=(-1)^{\frac{2(r i+j) k s+i(i-1) r s}{2}} \theta^{i r s} .
\end{aligned}
$$

Case II: $\Gamma=\mathbb{Z}_{2} \times \mathbb{Z}_{2} \times \mathbb{Z}_{2}=\langle x\rangle \times\langle y\rangle \times\langle z\rangle$. Let $\left\{e_{p, q, r}\right\}_{0 \leq p, q, r \leq 1}$ be the dual basis in $\mathbb{k}^{\Gamma}$, that is, $\left\langle e_{p, q, r}, x^{i} y^{j} z^{k}\right\rangle=\delta_{p, i} \delta_{q, j} \delta_{r, k}$ for $0 \leq i, j, k<2$. In this case,

$$
t \rightarrow e_{i, j, k}=e_{j, i, k}, \quad \theta(t)=\sum_{i j k p q r}(-1)^{k(p+q)} \xi_{1}^{j p} e_{i, j, k} \otimes e_{p, q, r}, \quad \sigma(t, t)=\sum \xi_{1}^{p q} e_{p, q, r} \sum \iota^{r} e_{p, q, r},
$$

where $\xi_{1}, \iota=0,1$. The Singer pair forms the Hopf algebra $H_{d: \xi_{1}, \iota}$. Furthermore,

$$
\begin{aligned}
& \tau_{t^{s}}\left(x^{f} y^{g} z^{h}, x^{l} y^{m} z^{n}\right)=\tau\left(x^{f} y^{g} z^{h}, x^{l} y^{m} z^{n}\right)\left(t^{s}\right)=\theta\left(t^{s}\right)\left(x^{f} y^{g} z^{h}, x^{l} y^{m} z^{n}\right) \\
& =\sum_{i j k p q r}(-1)^{k s(p+q)} \xi_{1}^{j p s}\left\langle e_{i, j, k} \otimes e_{p, q, r}, x^{f} y^{g} z^{h} \otimes x^{l} y^{m} z^{n}\right\rangle \\
& =(-1)^{s h(l+m)} \xi_{1}^{g l s} .
\end{aligned}
$$

We claim that $F \bowtie \Gamma$ is isomorphic to

$$
D_{8} \times \mathbb{Z}_{2}=G_{8}:=\left\langle x t, y, z \mid(x t)^{4}=y^{2}=z^{2}=1, y(x t) y=(x t)^{3},[x t, z]=[y, z]=1\right\rangle .
$$

Indeed, $x \triangleright t=y \triangleright t=z \triangleright t=t, x \triangleleft t=y, y \triangleleft t=x$, since $\triangleright$ is trivial and

$$
\begin{aligned}
\left\langle x \triangleleft t, e_{i, j, k}\right\rangle & =\left\langle x, t \rightarrow e_{i, j, k}\right\rangle=\left\langle x, e_{j, i, k}\right\rangle=\delta_{1, j} \delta_{0, i} \delta_{0, k}, \\
\left\langle y \triangleleft t, e_{i, j, k}\right\rangle & =\left\langle y, t \rightarrow e_{i, j, k}\right\rangle=\left\langle y, e_{j, i, k}\right\rangle=\delta_{0, j} \delta_{1, i} \delta_{0, k} .
\end{aligned}
$$

Similarly, we have $z \triangleleft t=z$. Then the claim follows by the following relations

$$
(1 \bowtie x)(t \bowtie 1)=(x \triangleright t) \bowtie(x \triangleleft t)=t \bowtie y, \quad(1 \bowtie y)(t \bowtie 1)=t \bowtie x,
$$

$$
(1 \bowtie z)(t \bowtie 1)=(z \triangleright t) \bowtie(z \triangleleft t)=t \bowtie z .
$$

Now we calculate $\omega(\sigma, \tau) \in Z^{3}\left(F \bowtie \Gamma, \mathbb{k}^{\times}\right)$.

(1) $H_{d: 1,1}$. Then $\sigma(t, t)=\sum e_{p, q, r} \sum e_{p, q, r}$ is trivial, that is, $\sigma_{x^{i} y^{j} z^{l}}\left(t^{r}, t^{s}\right)=1$. Let $a=t^{p} x^{i} y^{j} z^{k}$, $b=t^{q} x^{l} y^{m} z^{n}$ and $c=t^{r} x^{f} y^{g} z^{h}$. Then

$$
\begin{aligned}
& \omega(\tau, \sigma)(a, b, c)=\tau_{\pi(c)}(p(a) \triangleleft \pi(b), p(b)) \sigma_{p(a)}(\pi(b), p(b) \triangleright \pi(c)) \\
& =\tau_{t^{r}}\left(\left(x^{i} \triangleleft t^{q}\right)\left(y^{j} \triangleleft t^{q}\right) z^{k}, x^{l} y^{m} z^{n}\right) \\
& =\tau_{t^{r}}\left(x^{\frac{(i+j)+(-1)^{q}(i-j)}{2}} y^{\frac{(i+j)+(-1)^{q}(j-i)}{2}} z^{k}, x^{l} y^{m} z^{n}\right) \\
& =(-1)^{k r(l+m)} .
\end{aligned}
$$


(2) $H_{d: 1,-1}$. Since $\sigma(t, t)=\sum e_{p, q, r} \sum(-1)^{r} e_{p, q, r}$, it follows that $\sigma_{x^{i} y^{j} z^{l}}\left(t^{r}, t^{s}\right)=(-1)^{r s l}$. Then

$$
\begin{aligned}
& \omega(\tau, \sigma)(a, b, c)=\tau_{\pi(c)}(p(a) \triangleleft \pi(b), p(b)) \sigma_{p(a)}(\pi(b), p(b) \triangleright \pi(c)) \\
& =\tau_{t^{r}}\left(\left(x^{i} \triangleleft t^{q}\right)\left(y^{j} \triangleleft t^{q}\right) z^{k}, x^{l} y^{m} z^{n}\right) \sigma_{x^{i} y^{j} z^{l}}\left(t^{q}, t^{r}\right) \\
& =\tau_{t^{r}}\left(x^{\frac{(i+j)+(-1)^{q}(i-j)}{2}} y^{\frac{(i+j)+(-1)^{q}(j-i)}{2}} z^{k}, x^{l} y^{m} z^{n}\right) \sigma_{x^{i} y^{j} z^{l}}\left(t^{q}, t^{r}\right) \\
& =(-1)^{k r(l+m)}(-1)^{q r l}=(-1)^{(k l+k m+q l) r} .
\end{aligned}
$$

(3) $H_{d:-1,1}$. Since $\sigma(t, t)=\sum(-1)^{p q} e_{p, q, r} \sum e_{p, q, r}$, it follows that $\sigma_{x^{i} y^{j} z^{l}}\left(t^{r}, t^{s}\right)=(-1)^{r s i j}$. Then

$$
\begin{aligned}
& \omega(\tau, \sigma)(a, b, c)=\tau_{\pi(c)}(p(a) \triangleleft \pi(b), p(b)) \sigma_{p(a)}(\pi(b), p(b) \triangleright \pi(c)) \\
& =\tau_{t^{r}}\left(\left(x^{i} \triangleleft t^{q}\right)\left(y^{j} \triangleleft t^{q}\right) z^{k}, x^{l} y^{m} z^{n}\right) \sigma_{x^{i} y^{j} z^{l}}\left(t^{q}, t^{r}\right) \\
& =\tau_{t^{r}}\left(x^{\frac{(i+j)+(-1)^{q}(i-j)}{2}} y^{\frac{(i+j)+(-1)^{q}(j-i)}{2}} z^{k}, x^{l} y^{m} z^{n}\right) \sigma_{x^{i} y^{j} z^{l}}\left(t^{q}, t^{r}\right) \\
& =(-1)^{k r(l+m)+\frac{(i+j)+(-1)^{q}(j-i)}{2} l r}(-1)^{q r i j} .
\end{aligned}
$$

(4) $H_{d:-1,-1}$. Since $\sigma(t, t)=\sum(-1)^{p q} e_{p, q, r} \sum(-1)^{r} e_{p, q, r}$, it follows that $\sigma_{x^{i} y^{j} z^{l}}\left(t^{r}, t^{s}\right)=(-1)^{r s(i j+l)}$. Then

$$
\begin{aligned}
& \omega(\tau, \sigma)(a, b, c)=\tau_{\pi(c)}(p(a) \triangleleft \pi(b), p(b)) \sigma_{p(a)}(\pi(b), p(b) \triangleright \pi(c)) \\
& =\tau_{t^{r}}\left(\left(x^{i} \triangleleft t^{q}\right)\left(y^{j} \triangleleft t^{q}\right) z^{k}, x^{l} y^{m} z^{n}\right) \sigma_{x^{i} y^{j} z^{l}}\left(t^{q}, t^{r}\right) \\
& =\tau_{t^{r}}\left(x^{\frac{(i+j)+(-1)^{q}(i-j)}{2}} y^{\frac{(i+j)+(-1)^{q}(j-i)}{2}} z^{k}, x^{l} y^{m} z^{n}\right) \sigma_{x^{i} y^{j} z^{l}}\left(t^{q}, t^{r}\right) \\
& =(-1)^{k r(l+m)+\frac{(i+j)+(-1)^{q}(j-i)}{2} l r}(-1)^{q r(i j+l)} \text {. }
\end{aligned}
$$

Case III: $\Gamma=D_{8}=\left\langle x, y \mid x^{4}=y^{2}=1, y x=x^{-1} y\right\rangle$. Let $\left\{e_{p, q}\right\}_{0 \leq p \leq 3,0 \leq q \leq 1}$ be the dual basis in $\mathbb{k}^{\Gamma}$, that is, $\left\langle e_{p, q}, x^{i} y^{j}\right\rangle=\delta_{p, i} \delta_{q, j}$. Then $X=\sum_{p q}(-1)^{p} e_{p, q}, Y=\sum_{p q}(-1)^{q} e_{p, q}$ are group-like elements of order 2 and $\theta(t)=\sum_{i j p q} \xi^{j p} e_{i j} \otimes e_{p q}$, where $\xi^{4}=1$. Furthermore, we have

$$
\tau_{t^{s}}\left(x^{i} y^{j}, x^{k} y^{l}\right)=\xi^{j k s} .
$$

Case (B): The action is given by $t \rightarrow e_{p, q}=e_{-p, q}$ and $\sigma(t, t)=\sum(-1)^{k p+l q} e_{p, q}=X^{k} Y^{l}, 0 \leq k, l \leq$ 1. The Singer pair forms the Hopf algebra $H_{X^{k} Y^{l}}$. Moreover, $\xi$ is a primitive 4 -th root of unity.

We claim that $F \bowtie \Gamma$ is isomorphic to

$$
D_{8} \times \mathbb{Z}_{2}=G_{8}:=\left\langle x, t, y t \mid x^{4}=t^{2}=(y t)^{2}=1, t x=x^{-1} t,[x, y t]=[t, y]=1\right\rangle .
$$

Indeed, $x \triangleright t=y \triangleright t=t, x \triangleleft t=x^{-1}$ and $y \triangleleft t=y$, since $\triangleright$ is trivial and

$$
\begin{aligned}
& \left\langle x \triangleleft t, e_{i, j}\right\rangle=\left\langle x, t \rightarrow e_{i, j}\right\rangle=\left\langle x, e_{-i, j}\right\rangle=\delta_{1,-i} \delta_{0, j}, \\
& \left\langle y \triangleleft t, e_{i, j}\right\rangle=\left\langle y, t \rightarrow e_{i, j}\right\rangle=\left\langle y, e_{-i, j}\right\rangle=\delta_{0,-i} \delta_{1, j} .
\end{aligned}
$$

Then the claim follows by the following relations

$$
(1 \bowtie x)(t \bowtie 1)=t \bowtie x^{-1}, \quad(1 \bowtie y)(t \bowtie 1)=t \bowtie y .
$$

Now we calculate $\omega(\sigma, \tau) \in Z^{3}\left(F \bowtie \Gamma, \mathbb{k}^{\times}\right)$.

(1) $H_{B: 1}:=H_{X^{0} Y^{0}}$. Then $\sigma(t, t)=\sum e_{p, q}$ is trivial, that is, $\sigma_{x^{i} y^{j}}\left(t^{r}, t^{s}\right)=1$. Let $a=t^{q} x^{i} y^{j}, b=$ $t^{r} x^{k} y^{l}, c=t^{s} x^{m} y^{n}$ for some $0 \leq q, j, r, k, s, n<2,0 \leq i, k, m<4$. Then

$$
\begin{aligned}
& \omega(\tau, \sigma)(a, b, c)=\tau_{\pi(c)}(p(a) \triangleleft \pi(b), p(b)) \sigma_{p(a)}(\pi(b), p(b) \triangleright \pi(c)) \\
& =\tau_{t^{s}}\left(\left(x^{i} \triangleleft t^{r}\right) y^{j}, x^{k} y^{l}\right)=\tau_{t^{s}}\left(x^{(-1)^{r} i} y^{j}, x^{k} y^{l}\right)=\xi^{j k s} .
\end{aligned}
$$


(2) $H_{B: X}:=H_{X^{1} Y^{0}}$. Since $\sigma(t, t)=\sum(-1)^{p} e_{p, q}$, it follows that $\sigma_{x^{i} y^{j}}\left(t^{r}, t^{s}\right)=(-1)^{i r s}$. Then

$$
\begin{aligned}
& \omega(\tau, \sigma)(a, b, c)=\tau_{\pi(c)}(p(a) \triangleleft \pi(b), p(b)) \sigma_{p(a)}(\pi(b), p(b) \triangleright \pi(c)) \\
& =\tau_{t^{s}}\left(\left(x^{i} \triangleleft t^{r}\right) y^{j}, x^{k} y^{l}\right) \sigma_{x^{i} y^{j}}\left(t^{r}, t^{s}\right)=\tau_{t^{s}}\left(x^{(-1)^{r} i} y^{j}, x^{k} y^{l}\right)(-1)^{i r s} \\
& =(-1)^{i r s} \xi^{j k s} .
\end{aligned}
$$

Case (C): The action is given by $t \rightarrow e_{p, q}=e_{-p+q, q}$ and $\sigma_{k}(t, t)=\sum \mu^{k q} e_{p, q}$ for $k=0,1$, where $\xi=\mu^{-2 k}$ and $\mu$ is a primitive 8 -th root of unity. The Singer pair forms the Hopf algebra $H_{k}$.

We claim that $F \bowtie \Gamma$ is isomorphic to

$$
D_{16}=G_{3}:=\left\langle t y, y \mid(t y)^{8}=y^{2}=1,(t y) y=y(t y)^{-1}\right\rangle .
$$

Indeed, $x \triangleright t=y \triangleright t=t, x \triangleleft t=x^{-1}$ and $y \triangleleft t=x y$, since $\triangleright$ is trivial and

$$
\begin{aligned}
& \left\langle x \triangleleft t, e_{i, j}\right\rangle=\left\langle x, t \rightarrow e_{i, j}\right\rangle=\left\langle x, e_{-i+j, j}\right\rangle=\delta_{1,-i+j} \delta_{0, j}, \\
& \left\langle y \triangleleft t, e_{i, j}\right\rangle=\left\langle y, t \rightarrow e_{i, j}\right\rangle=\left\langle y, e_{-i+j, j}\right\rangle=\delta_{0,-i+j} \delta_{1, j} .
\end{aligned}
$$

Then the claim follows by the following relations

$$
\begin{gathered}
(1 \bowtie x)(t \bowtie 1)=(x \triangleright t) \bowtie(x \triangleleft t)=t \bowtie x^{-1}, \\
(1 \bowtie y)(t \bowtie 1)=(y \triangleright t) \bowtie(y \triangleleft t)=t \bowtie x y .
\end{gathered}
$$

Now we calculate $\omega(\sigma, \tau) \in Z^{3}\left(F \bowtie \Gamma, \mathbb{k}^{\times}\right)$.

(1) $H_{C: 1}:=H_{0}$. Since $\sigma(t, t)=\sum e_{p, q}$ and $\xi=1$, it follows that $\sigma$ and $\tau$ are trivial and hence $\omega(\tau, \sigma)$ is trivial.

(2) $H_{C: \sigma_{1}}:=H_{1}$. Since $\sigma(t, t)=\sum \omega^{q} e_{p, q}$, it follows that $\sigma_{x^{i} y^{j}}\left(t^{r}, t^{s}\right)=\mu^{j r s}$. Then

$$
\omega(\tau, \sigma)(a, b, c)=\xi^{j k s} \mu^{j r s}=\mu^{(r-2 k) j s} .
$$

Case IV: $\Gamma=Q_{8}=\left\langle x, y \mid x^{4}=1, y^{2}=x^{2}, y x=x^{-1} y\right\rangle$. Let $\left\{e_{p, q}\right\}_{0 \leq p \leq 3,0 \leq q \leq 1}$ be the basis of $\mathbb{k}^{\Gamma}$ dual to the basis $\left\{x^{p} y^{q}\right\}$. Then the action is given by $t \rightarrow e_{i, j}=e_{-i+j, j}, \sigma$ and $\tau$ are trivial and hence the 3-cocycle $\omega(\tau, \sigma)$ is trivial. The Singer pair forms the Hopf algebra $H_{E}$.

Recall that $x \triangleright t=y \triangleright t=t$. Since $(1 \bowtie x)(t \bowtie 1)=(x \triangleright t) \bowtie(x \triangleleft t)=t \bowtie x^{-1},(1 \bowtie y)(t \bowtie 1)=$ $(y \triangleright t) \bowtie(y \triangleleft t)=t \bowtie x y$, it follows that $F \bowtie \Gamma$ is isomorphic to

$$
S D_{16}=G_{2}:=\left\langle t y, t \mid(t y)^{8}=t^{2}=1, t(t y) t=(t y)^{3}\right\rangle .
$$

3.2. Isomorphism classes of Galois objects. In this subsection, we determine isomorphism classes of Galois objects of the Hopf algebras listed in [Ka, Table 1]. We first introduce some necessary materials that will be used later.

Lemma 3.1. Let $L$ be a subgroup of order 8 of $F \bowtie \Gamma$ such that $L \cap F=1$, where $F \bowtie \Gamma$ is one of the groups of order 16 mentioned in subsection 3.1. Then $L$ is isomorphic to one of the following groups:

Case I (a): $\mathbb{Z}_{4} \times \mathbb{Z}_{2}:\langle x\rangle \times\langle y\rangle,\langle x\rangle \times\left\langle t x^{3} y\right\rangle ; \quad D_{8}:\langle x t, y\rangle ; \quad Q_{8}:\langle x t, y t\rangle$.

Case I (b): $D_{8}:\langle x, t y\rangle,\langle x y, t y\rangle ; \quad \mathbb{Z}_{4} \times \mathbb{Z}_{2}:\langle x\rangle \times\langle y\rangle ; \quad \mathbb{Z}_{2} \times \mathbb{Z}_{2} \times \mathbb{Z}_{2}:\left\langle x^{2}\right\rangle \times\langle y\rangle \times\langle t x\rangle$.

Case I (c): $\mathbb{Z}_{4} \times \mathbb{Z}_{2}:\langle x\rangle \times\langle y\rangle,\langle t x\rangle \times\langle y\rangle$.

Case II: $\mathbb{Z}_{4} \times \mathbb{Z}_{2}:\langle x t\rangle \times\langle z\rangle ; \quad \mathbb{Z}_{2} \times \mathbb{Z}_{2} \times \mathbb{Z}_{2}:\langle x\rangle \times\langle y\rangle \times\langle z\rangle ; D_{8}:\langle x t, y z\rangle,\langle(x t) z, y\rangle$.

Case III (B): $\mathbb{Z}_{4} \times \mathbb{Z}_{2}:\langle x\rangle \times\langle y t\rangle ; \quad \mathbb{Z}_{2} \times \mathbb{Z}_{2} \times \mathbb{Z}_{2}:\left\langle x^{2}\right\rangle \times\langle x t\rangle \times\langle y t\rangle ;$

$D_{8}:\langle x, t(y t)\rangle=\langle x, y\rangle,\langle x(y t), t(y t)\rangle=\langle x(y t), y\rangle$.

Case III (C): $\mathbb{Z}_{8}:\langle t y\rangle ; \quad D_{8}:\langle x, y\rangle$.

Case IV: $\mathbb{Z}_{8}:\langle t y\rangle ; \quad Q_{8}:\langle x, y\rangle$. 
Proof. For Case I (a), $F \bowtie \Gamma \cong G_{7}$ with generators $x, y, t$. Then there are seven maximal subgroups, that is,

$$
\langle x\rangle \times\langle t\rangle, \quad\langle y, t\rangle, \quad\langle y x, t\rangle, \quad\langle x\rangle \times\langle y\rangle, \quad\langle x\rangle \times\langle y t x\rangle=\langle x\rangle \times\left\langle t x^{3} y\right\rangle, \quad\langle x t, y\rangle, \quad\langle x t, y t\rangle .
$$

For Case I (b), $F \bowtie \Gamma \cong D_{8} \times \mathbb{Z}_{2}$ with generators $x, t, y$. Then there are seven maximal subgroups, that is,

$$
\langle x\rangle \times\langle y\rangle, \quad\left\langle x^{2}\right\rangle \times\langle y\rangle \times\langle t\rangle, \quad\left\langle x^{2}\right\rangle \times\langle y\rangle \times\langle t x\rangle, \quad\langle x, t\rangle, \quad\langle x, t y\rangle, \quad\langle x y, t\rangle, \quad\langle x y, t y\rangle .
$$

For Case I (c), $F \bowtie \Gamma \cong G_{6}$ with generators $x, t, y$. Then there are three maximal subgroups, that is,

$$
\langle x\rangle \times\langle y\rangle, \quad\left\langle x^{2}\right\rangle \times\langle y\rangle \times\langle t\rangle, \quad\langle t x\rangle \times\langle y\rangle .
$$

For Case II, $F \bowtie \Gamma \cong D_{8} \times \mathbb{Z}_{2}$ with generators $x t, y, z$. Then there are seven maximal subgroups, that is,

$\langle x t\rangle \times\langle z\rangle, \quad\left\langle(x t)^{2}\right\rangle \times\langle z\rangle \times\langle y\rangle, \quad\left\langle(x t)^{2}\right\rangle \times\langle y(x t)\rangle \times\langle z\rangle, \quad\langle x t, y\rangle, \quad\langle x t, y z\rangle, \quad\langle(x t) z, y\rangle, \quad\langle(x t) z, y z\rangle$.

Observe that $(x t)^{2}=x y,(x t)^{3}=t x=y t$ and $(x t) z y z=x t y=t$.

For Case III (B), $F \bowtie \Gamma \cong D_{8} \times \mathbb{Z}_{2}$ with generators $x, t, y t$. Then there are seven maximal subgroups, that is,

$$
\langle x\rangle \times\langle y t\rangle, \quad\left\langle x^{2}\right\rangle \times\langle y t\rangle \times\langle t\rangle, \quad\left\langle x^{2}\right\rangle \times\langle y t\rangle \times\langle x t\rangle, \quad\langle x, t\rangle, \quad\langle x, t(y t)\rangle, \quad\langle x(y t), t\rangle, \quad\langle x(y t), t(y t)\rangle .
$$

For Case III (C), $F \bowtie \Gamma \cong D_{16}$ with generators $t y, y$. Then there are three maximal subgroups, that is,

$$
\langle t y\rangle, \quad\langle x, y\rangle, \quad\langle x, t x\rangle .
$$

For Case IV, $F \bowtie \Gamma \cong S D_{16}$ with generators $t y, t$. Then there are three maximal subgroups, that is,

$$
\langle t y\rangle, \quad\langle x, t\rangle, \quad\langle x, y\rangle .
$$

Consequently, the statements follow by direct computations.

The Schur multipliers of groups of order 8 are given by the following proposition.

Proposition $3.2([\underline{\mathrm{Kar}}])$. Let $L$ be a group of order 8 . Then the Schur multiplier $H^{2}\left(L, \mathbb{k}^{\times}\right)$is given as follow: $H^{2}\left(\mathbb{Z}_{8}, \mathbb{k}^{\times}\right)=H^{2}\left(Q_{8}, \mathbb{k}^{\times}\right)=0, H^{2}\left(\mathbb{Z}_{4} \times \mathbb{Z}_{2}, \mathbb{k}^{\times}\right) \cong H^{2}\left(D_{8}, \mathbb{k}^{\times}\right) \cong \mathbb{Z}_{2}, H^{2}\left(\mathbb{Z}_{2} \times \mathbb{Z}_{2} \times \mathbb{Z}_{2}, \mathbb{k}^{\times}\right) \cong$ $\mathbb{Z}_{2} \times \mathbb{Z}_{2} \times \mathbb{Z}_{2}$.

Remark 3.3. Let $G$ be a finite abelian group and $\sigma \in Z^{2}\left(G, \mathbb{k}^{\times}\right)$. Then the class of $\sigma$ is trivial, if and only if $\mathrm{k}_{\sigma}[G]$ is commutative, if and only if $\sigma$ is symmetric, that is, $\sigma(g, h)=\sigma(h, g)$ for any $g, h \in G$. Indeed, if the class of $\sigma$ is trivial, then $\mathbb{k}_{\sigma}[G] \cong \mathbb{k}[G]$ is commutative; if $\mathbb{k}_{\sigma}[G]$ is commutative, then irreducible representations of $\mathbb{k}_{\sigma}[G]$ are one-dimensional, hence there exists a morphism $\varphi: G \rightarrow \mathbb{k}^{\times}$ such that $\varphi\left(g \cdot{ }_{\sigma} h\right)=\varphi(g) \varphi(h)$, that is, $\varphi(g h)^{-1} \varphi(g) \varphi(h)=\sigma(g, h)$. Obviously, $\mathbb{k}_{\sigma}[G]$ is commutative if and only if $\sigma$ is symmetric.

Now we describe explicitly $H^{2}\left(\mathbb{Z}_{2} \times \mathbb{Z}_{2} \times \mathbb{Z}_{2}, \mathbb{k}^{\times}\right)$, which was determined in [BPW].

Proposition 3.4. ([BPW, Proposition 4.10]) A set of cocycles $\sigma: \mathbb{Z}_{2} \times \mathbb{Z}_{2} \times \mathbb{Z}_{2} \rightarrow \mathbb{k}^{\times}$which represents $H^{2}\left(\mathbb{Z}_{2} \times \mathbb{Z}_{2} \times \mathbb{Z}_{2}, \mathbb{k}^{\times}\right)$is given by

$$
\sigma\left(g_{1}^{i_{1}} g_{2}^{i_{2}} g_{3}^{i_{3}}, g_{1}^{j_{1}} g_{2}^{j_{2}} g_{3}^{j_{3}}\right)=(-1)^{a_{12} i_{1} j_{2}+a_{13} i_{1} j_{3}+a_{23} i_{2} j_{3}},
$$

where $g_{1}, g_{2}, g_{3}$ are generators of $\mathbb{Z}_{2} \times \mathbb{Z}_{2} \times \mathbb{Z}_{2}$ and $i_{l}, j_{k}, a_{m n} \in\{0,1\}$ for $1 \leq k, l, m, n \leq 3$. 
Let $\alpha \in H^{2}\left(\mathbb{Z}_{2} \times \mathbb{Z}_{2} \times \mathbb{Z}_{2}, \mathbb{k}^{\times}\right)$. Then by Proposition 3.4, $\alpha$ is uniquely determined by a sequence $\left(a_{12}, a_{13}, a_{23}\right)$. Hence $\left(a_{12}, a_{13}, a_{23}\right)$ can be used to label $\alpha$.

Let $G \cong \mathbb{Z}_{m_{1}} \times \cdots \times \mathbb{Z}_{m_{n}}:=\left\langle g_{1}\right\rangle \times \cdots \times\left\langle g_{n}\right\rangle$. An explicit and unified formula of the normalized 3 -cocycle on $G$ is determined in [HLYY]. Let $A$ be the set of all sequences

$$
\underline{\mathbf{a}}:=\left(a_{1}, \ldots, a_{l}, \ldots, a_{n}, a_{12}, \ldots, a_{i j}, \ldots, a_{n-1, n}, a_{123}, \ldots, a_{r s t}, \ldots, a_{n-2, n-1, n}\right)
$$

such that $0 \leq a_{l}<m_{l}, 0 \leq a_{i j}<\left(m_{i}, m_{j}\right), 0 \leq a_{r s t}<\left(m_{r}, m_{s}, m_{t}\right)$ for $1 \leq l \leq n, 1 \leq i<j \leq n, 1 \leq$ $r<s<t \leq n$ where $a_{i j}$ and $a_{r s t}$ are ordered by the lexicographic order. Here $\left(s_{1}, \ldots, s_{t}\right)$ denotes the greatest common divisor of $s_{1}, \ldots, s_{t} \in \mathbb{Z}_{+}$.

Let $\nu_{n}$ be a primitive $n$-th root of unity for any positive integer $n$, and $\left[\frac{r}{n}\right]$ the largest integer less than $\frac{r}{n}$ for $r \in \mathbb{Z}$. For any $\underline{\mathbf{a}} \in A$, define a $\mathbb{Z} G$-module morphism:

$$
\begin{aligned}
& \omega_{\underline{\mathbf{a}}}: G \times G \times G \rightarrow \mathbb{k}^{\times} \\
& {\left[g_{1}^{i_{1}} \cdots g_{n}^{i_{n}}, g_{1}^{j_{1}} \cdots g_{n}^{j_{n}}, g_{1}^{k_{1}} \cdots g_{n}^{k_{n}}\right] \mapsto \prod_{l=1}^{n} \nu_{m_{l}}^{a_{l} i_{l}\left[\frac{j_{l}+k_{l}}{m_{l}}\right]} \prod_{1 \leq s<t \leq n} \nu_{m_{t}}^{a_{s t} i_{t}\left[\frac{j_{s}+k_{s}}{m_{s}}\right]} \prod_{1 \leq r<s<t \leq n} \nu_{\left(m_{r}, m_{s}, m_{t}\right)}^{-a_{r s t} k_{r} j_{s} i_{t}} .}
\end{aligned}
$$

Proposition 3.5. [HLYY, Proposition 3.8] $\left\{\omega_{\underline{\mathbf{a}}} \mid \underline{\mathbf{a}} \in A\right\}$ is a complete set of representatives of normalized 3-cocycles on $G$.

Remark 3.6. If $G \cong \mathbb{Z}_{N}:=\langle g\rangle$, then a complete set of representative of $H^{3}\left(G, \mathbb{k}^{\times}\right)$is given by

$$
\omega_{a}\left(g^{i}, g^{j}, g^{k}\right)=\nu_{N}^{a k\left[\frac{i+j}{N}\right]}, \quad 0 \leq a, i, j, k<N .
$$

If $G \cong \mathbb{Z}_{N} \times \mathbb{Z}_{M}:=\langle g\rangle \times\langle h\rangle$, then a complete set of representative of $H^{3}\left(G, \mathbb{k}^{\times}\right)$is given by

$$
\omega_{\underline{\mathbf{a}}}\left(g^{i} h^{j}, g^{k} h^{l}, g^{m} h^{n}\right)=\nu_{M}^{a_{1} i\left[\frac{k+m}{M}\right]} \nu_{N}^{a_{2} j\left[\frac{l+n}{N}\right]} \nu_{N}^{a_{12} j\left[\frac{k+m}{M}\right]}, \quad 0 \leq i, k, m<M, 0 \leq j, l, n<N,
$$

where $\underline{\mathbf{a}}:=\left(a_{1}, a_{2}, a_{12}\right) \in A$.

Let $H=\left(\mathbb{k}^{\Gamma \rho, \theta} \# \rightarrow, \sigma \mathbb{k}[F]\right)^{*}$ be a Hopf algebra listed in [Ka, Table 1]. Now we compute the number of Galois objects of $H$ up to isomorphism. By Proposition 2.11, we proceed this by computing the number of isomorphism classes of fiber functors $(L, \alpha)$ on $\mathcal{C}(F \bowtie \Gamma, \omega(\tau, \sigma), F, 1)$.

From Theorem 2.2, condition (3) forces $L \cap F=1$; then condition (2) implies $L$ is a normal subgroup of order 8 and $F \bowtie \Gamma=L F$ is an exact factorization; furthermore, fiber functors $(L, \alpha),\left(L^{\prime}, \alpha^{\prime}\right)$ are isomorphic if and only if $L=L^{\prime}$ and there exists an element $g \in G$ such that the class $\alpha^{-1} \alpha^{\prime g} \Omega_{g}$ is trivial in $H^{2}\left(L, \mathbb{k}^{\times}\right)$.

Consequently, it suffices to find all subgroups $L$ of order 8 satisfying the conditions

$$
L \cap F=1 \text { and the class of }\left.\omega(\tau, \sigma)\right|_{L \times L \times L} \text { is trivial, }
$$

and then determine all isomorphism classes of pairs $(L, \alpha)$ for $[\alpha] \in H^{2}\left(L, \mathbb{k}^{\times}\right)$. In what follows, unless specified otherwise, $L$ denotes a subgroup of order 8 satisfying the condition (6) and $\omega:=\omega(\tau, \sigma)$.

Remark 3.7. If $L \cong \mathbb{Z}_{4} \times \mathbb{Z}_{2}$ or $D_{8}$, then by Proposition $3.2, H^{2}\left(L, \mathbb{k}^{\times}\right) \cong \mathbb{Z}_{2}$. Let $[\alpha]$ be a generator of $H^{2}\left(L, \mathbb{k}^{\times}\right)$. Then by Theorem $2.2,(L, 1)$ and $(L, \alpha)$ are isomorphic if and only if

- the class of $\left.\alpha \Omega_{g}\right|_{L \times L}$ is trivial in $H^{2}\left(L, \mathbb{k}^{\times}\right)$for some $g \in G$.

Furthermore, if $L \cong \mathbb{Z}_{4} \times \mathbb{Z}_{2}$, by Remark 3.3, they are isomorphic if and only if $\left.\Omega_{g}\right|_{L \times L}$ is not symmetric.

Lemma 3.8. Up to isomorphism, $\left(H_{a: 1}\right)^{*}$ has one or two Galois objects, and $\left(H_{a: y}\right)^{*}$ has four Galois objects.

Proof. By Lemma 3.1, the possibilities of $L$ are $\langle x\rangle \times\langle y\rangle \cong \mathbb{Z}_{4} \times \mathbb{Z}_{2},\langle x\rangle \times\left\langle t x^{3} y\right\rangle \cong \mathbb{Z}_{4} \times \mathbb{Z}_{2},\langle x t, y\rangle \cong D_{8}$, $\langle x t, y t\rangle \cong Q_{8}$. Let $a=t^{q} x^{i} y^{j}, b=t^{r} x^{k} y^{l}$ and $c=t^{s} x^{m} y^{n}$ for some $q, r, s, j, l, n \in \mathbb{Z}_{2}, i, k, m \in \mathbb{Z}_{4}$. 
For $H_{a: 1}$, we have $\omega(a, b, c)=(-1)^{j k s}$. We first claim that $L \neq\langle x\rangle \times\left\langle t x^{3} y\right\rangle$ or $\langle x t, y\rangle$. Indeed, consider the restriction of $\omega$ on the subgroup $\left\langle t x^{3} y\right\rangle \cong \mathbb{Z}_{2}$ of $L$, we have

$$
\omega\left(\left(t x^{3} y\right)^{i},\left(t x^{3} y\right)^{j},\left(t x^{3} y\right)^{k}\right)=(-1)^{i j k}=(-1)^{k\left[\frac{i+j}{2}\right]}, \quad i, j, k \in\{0,1\} ;
$$

then by Remark 3.6, the class of $\left.\omega\right|_{\left\langle t x^{3} y\right\rangle \times\left\langle t x^{3} y\right\rangle \times\left\langle t x^{3} y\right\rangle}$ is not trivial in $H^{3}\left(\mathbb{Z}_{2}, \mathbb{k}^{\times}\right)$, which implies that the class $\left.\omega\right|_{L \times L \times L}$ is non-trivial and hence the claim follows.

Assume that $L=\langle x\rangle \times\langle y\rangle \cong \mathbb{Z}_{4} \times \mathbb{Z}_{2}$. Clearly, $\left.\omega\right|_{L \times L \times L}=1$. By Proposition 3.3, $H^{2}\left(\mathbb{Z}_{4} \times \mathbb{Z}_{2}, \mathbb{k}^{\times}\right) \cong$ $\mathbb{Z}_{2}$. Let $\{[\alpha]\}$ be a set of representative of $H^{2}\left(\mathbb{Z}_{4} \times \mathbb{Z}_{2}, \mathbb{k}^{\times}\right)$. We claim that $(L, 1)$ and $(L, \alpha)$ are isomorphic. By definition,

$$
\Omega_{t}\left(x^{k} y^{l}, x^{m} y^{n}\right)=\frac{\omega\left(t x^{k} y^{l} t, t x^{m} y^{n} t, t\right) \omega\left(t, x^{k} y^{l}, x^{m} y^{n}\right)}{\omega\left(t x^{k} y^{l} t, t, x^{m} y^{n}\right)}=(-1)^{l m} .
$$

It is easy to see that the class of $\left.\Omega_{t}\right|_{L \times L}$ is not symmetric. Then the claim follows by Remark 3.7 .

Assume that $L=\langle x t, y t\rangle \cong Q_{8}$. Then by Proposition 3.2, $H^{2}\left(Q_{8}, \mathbb{k}^{\times}\right)=0$. Hence there exists at most one Galois object for $L=\langle x t, y t\rangle$, depending on whether the class of $\left.\omega\right|_{L \times L \times L}$ is trivial or not. Consequently, up to isomorphism there exists one or two trivial Galois objects for $\left(H_{a: 1}\right)^{*}$.

For $H_{a: y}$, we have $\omega(a, b, c)=(-1)^{j(k+r) s}$. Assume that $L=\langle x\rangle \times\left\langle t x^{3} y\right\rangle \cong \mathbb{Z}_{4} \times \mathbb{Z}_{2}$. We claim that $\left.\omega\right|_{L \times L \times L}$ is trivial in $H^{3}\left(L, \mathbb{k}^{\times}\right)$. Observe that $x^{i}\left(t x^{3} y\right)^{j}=t^{j} x^{3 j+i} y^{j}$. Let $\beta:=t x^{3} y$ for short. By Remark 3.6, there is a 2-chain $\varphi: L \times L \rightarrow \mathbb{k}^{\times}$and a sequence $\underline{\mathbf{a}}:=\left(a_{1}, a_{2}, a_{12}\right)$ such that

$$
\left.d^{2}(\varphi) \omega\right|_{L \times L \times L}\left(x^{i} \beta^{j}, x^{k} \beta^{l}, x^{m} \beta^{n}\right)=\omega_{\underline{\mathbf{a}}}\left(x^{i} \beta^{j}, x^{k} \beta^{l}, x^{m} \beta^{n}\right)=\nu_{4}^{a_{1} i\left[\frac{k+m}{4}\right]} \nu_{2}^{a_{2} j\left[\frac{l+n}{2}\right]} \nu_{2}^{a_{12} j\left[\frac{k+m}{4}\right]} .
$$

Since $\left.\omega\right|_{P \times P \times P}=1$ for $P=\langle x\rangle$ and $\langle\beta\rangle$, it follows that $a_{1}=0=a_{2}$. Now consider the subgroup $Q:=\langle t x y\rangle=\left\langle x^{2} \beta\right\rangle$ of $L$, clearly $\left.\omega\right|_{Q \times Q \times Q}=1$. Then

$$
d^{2}(\varphi)(t x y, t x y, t x y)=\omega_{\underline{\mathbf{a}}}(t x y, t x y, t x y)=\nu_{2}^{a_{12}}=(-1)^{a_{12}} .
$$

Meanwhile, $d^{2}(\varphi)(t x y, t x y, t x y)=\frac{\varphi(t x y, 1)}{\varphi(1, t x y)}$, and $\varphi(t x y, 1)=\varphi(1, t x y)=\varphi(1,1)$, which implies $a_{12}=0$ and hence the claim follows.

Now we show that $(L, 1)$ and $(L, \alpha)$ are isomorphic. Observe that $x t=t x$ and $y^{l} t=t x^{2 l} y^{l}$ for $0 \leq l \leq 1$. By definition,

$$
\begin{aligned}
\Omega_{t}\left(x^{k} \beta^{l}, x^{m} \beta^{n}\right) & =\frac{\omega\left(t^{l+1} x^{k+3 l} y^{l} t, t^{n+1} x^{m+3 n} y^{n} t, t\right) \omega\left(t, t^{l} x^{k+3 l} y^{l}, t^{n} x^{3 n+m} y^{n}\right)}{\omega\left(t^{l+1} x^{k+3 l} y^{l} t, t, t^{n} x^{3 n+m} y^{n}\right)} \\
& =\frac{\omega\left(t^{l} x^{k+l} y^{l}, t^{n} x^{m+n} y^{n}, t\right) \omega\left(t, t^{l} x^{k+3 l} y^{l}, t^{n} x^{3 n+m} y^{n}\right)}{\omega\left(t^{l} x^{k+l} y^{l}, t, t^{n} x^{3 n+m} y^{n}\right)}=(-1)^{(n+m) l} .
\end{aligned}
$$

It is easy to see that $\Omega_{t}$ is not symmetric. Then the claim follows by Remark 3.7.

Assume that $L=\langle x\rangle \times\langle y\rangle \cong \mathbb{Z}_{4} \times \mathbb{Z}_{2},\langle x t, y\rangle \cong D_{8}$ or $\langle x t, y t\rangle \cong Q_{8}$. It is easy to see that $\left.\omega\right|_{L \times L \times L}=1$. Now we determine isomorphism classes of fiber functors. If $L=\langle x\rangle \times\langle y\rangle$, then $\Omega_{t}\left(x^{k} y^{l}, x^{m} y^{n}\right)=(-1)^{l m}$, which is not symmetric, and hence there is only one isomorphism class. If $L=\langle x t, y t\rangle \cong Q_{8}$, then by Proposition 3.2 , there is only one isomorphism class. If $L=\langle x t, y\rangle \cong D_{8}$, then

$$
\Omega_{t}\left((x t)^{k} y^{l},(x t)^{m} y^{n}\right)=\frac{\omega\left(t^{k} x^{k+2 l} y^{l}, t^{m} x^{m+2 n} y^{n}, t\right) \omega\left(t, t^{k} x^{k} y^{l}, t^{m} x^{m} y^{n}\right)}{\omega\left(t^{k} x^{k+2 l} y^{l}, t, t^{m} x^{m} y^{n}\right)}=(-1)^{l m}
$$

which implies that the class of $\left.\Omega_{t}\right|_{L \times L}$ is not trivial in $H^{2}\left(L, \mathbb{k}^{\times}\right)$, and hence by Remark 3.7 there is only one isomorphism. Indeed if there exists a morphism $f: L \rightarrow \mathbb{k}^{\times}$such that $d(f)=\left.\Omega_{t}\right|_{L \times L}$, then $f\left((x t)^{k}\right)=f(x t)^{k}$ and $f\left((x t)^{4}\right)=f\left(y^{2}\right)=f(y)^{2}=1$. Since $\Omega_{t}(x t, y)=1, f(y(x t))=f\left((x t)^{3} y\right)=$ $f(x t)^{3} f(y)$, while then $-1=\Omega_{t}(y, x t)=f(y) f(x t) f(y(x t))^{-1}=f(y)^{2} f(x t)^{4}=1$, impossible.

Therefore, up to isomorphism there are four Galois objects for $\left(H_{a: y}\right)^{*}$. 
Remark 3.9. From the proof of Lemma 3.8, if the class of $\left.\omega\right|_{L \times L \times L}$ for $L=\langle x t, y t\rangle \cong Q_{8}$ is trivial, then $\left(H_{a: 1}\right)^{*}$ has two Galois objects; otherwise $\left(H_{a: 1}\right)^{*}$ has only one Galois object.

Lemma 3.10. Up to isomorphism, $\left(H_{b: 1}\right)^{*}$ has one or two Galois objects, $\left(H_{b: y}\right)^{*}$ and $\left(H_{b: x^{2} y}\right)^{*}$ have seven Galois objects.

Proof. By Lemma 3.1, the possibilities of $L$ are $\langle x, t y\rangle \cong D_{8},\langle x y, t y\rangle \cong D_{8},\langle x\rangle \times\langle y\rangle \cong \mathbb{Z}_{4} \times \mathbb{Z}_{2}$, $\left\langle x^{2}\right\rangle \times\langle y\rangle \times\langle t x\rangle \cong \mathbb{Z}_{2} \times \mathbb{Z}_{2} \times \mathbb{Z}_{2}$. Let $a=t^{q} x^{i} y^{j}, b=t^{r} x^{k} y^{l}$ and $c=t^{s} x^{m} y^{n}$ for some $q, r, s, j, l, n \in$ $\mathbb{Z}_{2}, i, k, m \in \mathbb{Z}_{4}$.

For $H_{b: 1}$, we have $\omega(a, b, c)=(-1)^{j k s}$. If $L=\langle x, t y\rangle$ or $\left\langle x^{2}\right\rangle \times\langle y\rangle \times\langle t x\rangle$, then the restriction of $\omega$ on the subgroup $\left\langle t x^{3} y\right\rangle$ is not trivial in $H^{3}\left(\left\langle t x^{3} y\right\rangle, \mathbb{k}^{\times}\right)$and hence $\left.\omega\right|_{L \times L \times L}$ is not trivial in $H^{3}\left(L, \mathbb{k}^{\times}\right)$.

Assume that $L=\langle x y, t y\rangle \cong D_{8}$. Then a direct computation shows that the class of $\left.\Omega_{t}\right|_{L \times L}$ is non-trivial. If the class of $\left.\omega\right|_{L \times L \times L}$ is non-trivial, then there is no Galois object; otherwise there is one Galois object by Remark 3.7. Hence there is at most one Galois object in this case.

Assume that $L=\langle x, y\rangle \cong \mathbb{Z}_{4} \times \mathbb{Z}_{2}$. Clearly, $\left.\omega\right|_{L \times L \times L}=1$. By definition, $\Omega_{t}\left(x^{k} y^{l}, x^{m} y^{n}\right)=(-1)^{l m}$, which is not symmetric. Then by Remark $3.7,(L, \alpha)$ and $(L, 1)$ are isomorphic, where $[\alpha]$ generates $H^{2}\left(L, \mathbb{k}^{\times}\right)$. Consequently, $\left(H_{b: 1}\right)^{*}$ has one or two Galois objects up to isomorphism.

For $H_{b: y}$ and $H_{b: x^{2} y}, \omega(a, b, c)=(-1)^{j(k+r) s}$ and $(-1)^{(j k+j r+i r) s}$, respectively. We claim that $L \neq$ $\langle x, t y\rangle$ or $\langle x y, t y\rangle$. Indeed, consider the subgroup $\langle t y\rangle \cong \mathbb{Z}_{2}$ of $L$, we have

$$
\omega\left((t y)^{i},(t y)^{j},(t y)^{k}\right)=(-1)^{k\left[\frac{i+j}{2}\right]} ;
$$

then by Remark 3.6, the class of $\left.\omega\right|_{\langle t y\rangle \times\langle t y\rangle \times\langle t y\rangle}$ is non-trivial, which implies that $\left.\omega\right|_{L \times L \times L}$ is non-trivial.

Assume that $L=\langle x\rangle \times\langle y\rangle \cong \mathbb{Z}_{4} \times \mathbb{Z}_{2}$ or $\left\langle x^{2}\right\rangle \times\langle y\rangle \times\langle t x\rangle \cong \mathbb{Z}_{2} \times \mathbb{Z}_{2} \times \mathbb{Z}_{2}$. It is easy to see that $\left.\omega\right|_{L \times L \times L}=1$. Now we determine the isomorphism classes of the fiber functors. If $L=\langle x\rangle \times\langle y\rangle$, then there is one isomorphism class since $\left.\Omega_{t}\right|_{L \times L}$ is not symmetric. Now we focus on $L=\left\langle x^{2}\right\rangle \times\langle y\rangle \times\langle t x\rangle$. For $H_{b: y}$, a direct computation shows $\Omega_{t \pi}=\Omega_{t}$ for all $\pi \in L$ and $\Omega_{t}\left(\left(x^{2}\right)^{i} y^{j}(t x)^{k},\left(x^{2}\right)^{l} y^{m}(t x)^{n}\right)=$ $(-1)^{j n}$. Let $(L, \alpha)$ and $(L, \beta)$ be two fiber functors, where $[\alpha],[\beta] \in H^{2}\left(L, \mathbb{k}^{\times}\right)$are determined by the sequences $\left(a_{12}, a_{13}, a_{23}\right)$ and $\left(b_{12}, b_{13}, b_{23}\right)$ by Proposition 3.4 , respectively. By Theorem 2.2 and Remark 3.3. they are isomorphic if and only if there exists $g \in G$ such that the class of $\alpha \beta^{g} \Omega_{g}$ is trivial, if and only if $\alpha \beta^{g} \Omega_{g}$ is symmetric. Since

$$
\alpha \beta^{t} \Omega_{t}\left(\left(x^{2}\right)^{i} y^{j}(t x)^{k},\left(x^{2}\right)^{l} y^{m}(t x)^{n}\right)=(-1)^{\left(a_{12}+b_{12}\right) i m+\left(a_{13}+b_{13}\right) i n+\left(a_{23}+b_{23}+1\right) j n+b_{12} k m+b_{13} k n},
$$

$\alpha \beta^{t} \Omega_{t}$ is symmetric if and only if $b_{12}=a_{12}=0, b_{13}+a_{13} \equiv 0 \bmod 2$ and $a_{23}+b_{23}+1 \equiv 0 \bmod 2$, which means $(0,0,0)$ and $(0,0,1)$ are isomorphic, $(0,1,0)$ and $(0,1,1)$ are isomorphic. For other $t l$ $(l \in L)$, the results on equivalence classes are the same. Consequently, there are seven Galois objects for $\left(H_{b: y}\right)^{*}$. For $H_{b: x^{2} y}$, the proof follows the same lines.

Remark 3.11. From the proof of Lemma 3.10, if $\left.\omega\right|_{L \times L \times L}$ for $L=\langle x y, t y\rangle \cong D_{8}$ is trivial, then $\left(H_{b: 1}\right)^{*}$ has only one Galois object; otherwise $\left(H_{b: 1}\right)^{*}$ has two Galois objects.

Lemma 3.12. Up to isomorphism, $\left(H_{c: \sigma_{0}}\right)^{*}$ has two Galois objects and $\left(H_{c: \sigma_{1}}\right)^{*}$ only has trivial Galois object.

Proof. By Lemma 3.1, the possibilities of $L$ are $\langle x\rangle \times\langle y\rangle \cong \mathbb{Z}_{4} \times \mathbb{Z}_{2},\langle t x\rangle \times\langle y\rangle \cong \mathbb{Z}_{4} \times \mathbb{Z}_{2}$. Let $a=t^{q} x^{i} y^{j}, b=t^{r} x^{k} y^{l}$ and $c=t^{s} x^{m} y^{n}$ for some $q, r, s, j, l, n \in \mathbb{Z}_{2}, i, k, m \in \mathbb{Z}_{4}$.

For $H_{c: \sigma_{0}}, \omega(a, b, c)=(-1)^{\frac{2(r i+j) k s+i(i-1) r s}{2}}$. Assume that $L=\langle t x\rangle \times\langle y\rangle \cong \mathbb{Z}_{4} \times \mathbb{Z}_{2}$. We first show that the class of $\left.\omega\right|_{L \times L \times L}$ is trivial. Observe that $(t x)^{i} y^{j}=t^{i} x^{i} y^{\left[\frac{i}{2}\right]+j}$; then direct computations show that $\left.\omega\right|_{P \times P \times P}=1$ if $P=\langle t x\rangle,\langle y\rangle$ or $\left\langle(t x)^{2} y\right\rangle=\left\langle x^{2}\right\rangle$; following the same line as the proof of the case $H_{a: y}$ in Lemma 3.8, the claim follows. Now we show that $(L, 1)$ and $(L, \alpha)$ are isomorphic, 
where $[\alpha]$ generates $H^{2}\left(\mathbb{Z}_{4} \times \mathbb{Z}_{2}, \mathbb{k}^{\times}\right)$. Let $\beta:=t x$ for short. Observe that $t y=y t, x^{k} t=t x^{k} y^{k}$ and $\beta^{k} t=t^{k+1} x^{k} y^{\left[\frac{k}{2}\right]+k}=t \beta^{k} y^{k}$. By definition,

$$
\begin{aligned}
\Omega_{t}\left(\beta^{k} y^{l}, \beta^{m} y^{n}\right) & =\frac{\omega\left(\beta^{k} y^{k+l}, \beta^{m} y^{m+n}, t\right) \omega\left(t, \beta^{k} y^{l}, \beta^{m} y^{n}\right)}{\omega\left(\beta^{k} y^{k+l}, t, \beta^{m} y^{n}\right)} \\
& =\frac{\omega\left(t^{k} x^{k} y^{\left[\frac{k}{2}\right]+k+l}, t^{m} x^{m} y^{\left[\frac{m}{2}\right]+m+n}, t\right) \omega\left(t, t^{k} x^{k} y^{\left[\frac{k}{2}\right]+l}, t^{m} x^{m} y^{\left[\frac{m}{2}\right]+n}\right)}{\omega\left(t^{k} x^{k} y^{\left[\frac{k}{2}\right]+k+l}, t, t^{m} x^{m} y^{\left[\frac{m}{2}\right]+n}\right)} \\
& =(-1)^{m k+\left[\frac{k}{2}\right]+k+l} .
\end{aligned}
$$

It is easy to see that $\Omega_{t}$ is not symmetric. Hence by Remark 3.7 $(L, 1)$ and $(L, \alpha)$ are isomorphic.

Assume that $L=\langle x\rangle \times\langle y\rangle \cong \mathbb{Z}_{4} \times \mathbb{Z}_{2}$. Clearly, $\left.\omega\right|_{L \times L \times L}=1$. We claim that $(L, 1)$ and $(L, \alpha)$ are isomorphic. Indeed, $\Omega_{t}\left(x^{k} y^{l}, x^{m} y^{n}\right)=(-1)^{(k+l) m}$, which is not symmetric, hence the claim follows by Remark 3.7. Consequently, there are two Galois objects up to isomorphism for $\left(H_{c: \sigma_{0}}\right)^{*}$.

For $H_{c: \sigma_{1}}$, we have $\omega(a, b, c)=(-1)^{\frac{2(r i+j) k s+i(i-1) r s}{2}} \theta^{i r s}$. We first claim that $L \neq\langle t x\rangle \times\langle y\rangle$. Indeed, it suffices to show that the restriction of $\omega$ on the subgroup $\langle t x\rangle \cong \mathbb{Z}_{4}$ is non-trivial. By Remark 3.6, there is a 2-cochain $\varphi:\langle t x\rangle \times\langle t x\rangle \rightarrow \mathbb{k}^{\times}$such that $\omega d^{2}(\varphi)=\omega_{a}$ for some $0 \leq a<4$. Observe that $\left(\omega d^{2}(\varphi)\right)\left(t x,(t x)^{j}, t x\right)=\nu_{4}^{a}$ if $j=3$ otherwise it equals to 1 . Then $\nu_{4}^{a}=-\theta \frac{\varphi\left((t y)^{3}, t y\right)}{\varphi\left(t y,(t y)^{3}\right)}=(-\theta)^{2}=-1$, that is, the class of $\left.\omega\right|_{\langle t x\rangle \times\langle t x\rangle \times\langle t x\rangle}$ is non-trivial, which implies that the claim follows.

Assume that $L=\langle x\rangle \times\langle y\rangle \cong \mathbb{Z}_{4} \times \mathbb{Z}_{2}$. Clearly, $\left.\omega\right|_{L \times L \times L}=1$. Since $\left.\Omega_{t}\right|_{L \times L}$ is not symmetric, it follows by Remark 3.7 that $(L, 1)$ and $(L, \alpha)$ are isomorphic, where $[\alpha]$ represents $H^{2}\left(L, \mathbb{k}^{\times}\right) \cong \mathbb{Z}_{2}$. Consequently, there are only trivial Galois objects for $\left(H_{c: \sigma_{1}}\right)^{*}$.

Lemma 3.13. Up to isomorphism, $\left(H_{d:-1,1}\right)^{*} \cong H_{d:-1,1}$ has four Galois objects, $\left(H_{d:-1,-1}\right)^{*} \cong H_{c: \sigma_{0}}$ and $\left(H_{d: 1,-1}\right)^{*} \cong H_{b: 1}$ have five Galois objects, the number of Galois objects of $\left(H_{d: 1,1}\right)^{*} \cong H_{d: 1,1}$ ranges from six to eight.

Proof. By Lemma 3.1, the possibilities of $L$ are $\langle x t\rangle \times\langle z\rangle \cong \mathbb{Z}_{4} \times \mathbb{Z}_{2},\langle x\rangle \times\langle y\rangle \times\langle z\rangle \cong \mathbb{Z}_{2} \times \mathbb{Z}_{2} \times \mathbb{Z}_{2}$, $\langle x t, y z\rangle \cong D_{8},\langle(x t) z, y\rangle \cong D_{8}$.

For $H_{d:-1,1}$ and $H_{d: 1,-1}$, we claim that $L=\langle x\rangle \times\langle y\rangle \times\langle z\rangle \cong \mathbb{Z}_{2} \times \mathbb{Z}_{2} \times \mathbb{Z}_{2}$. Indeed, take $H_{d:-1,1}$ for example, if $L=\langle x t\rangle \times\langle z\rangle$ or $\langle(x t) z, y\rangle$, then consider the restriction of $\omega$ on the subgroup $\langle(x t) z\rangle \cong \mathbb{Z}_{4}$, by Remark 3.6, there is a 2-cochain $\varphi: \mathbb{Z}_{4} \times \mathbb{Z}_{4} \rightarrow \mathbb{k}^{\times}$such that $\omega d^{2}(\varphi)=\omega_{a}$ for some $0 \leq a<4$. A direct computation shows that $a=2$, that is, the class of $\omega \mid\langle(x t) z\rangle \times\langle(x t) z\rangle \times\langle(x t) z\rangle$ is non-trivial in $H^{3}\left(\mathbb{Z}_{4}, \mathbb{k}^{\times}\right)$and hence the claim follows. If $L=\langle x t, y z\rangle$, then similar computations as before show that the class of $\omega$ is non-trivial when restricted to the subgroup $\langle x t\rangle$. Clearly, $\left.\omega\right|_{L \times L \times L}=1$ if $L=\langle x\rangle \times\langle y\rangle \times\langle z\rangle$. Therefore, the claim follows.

Now we determine the isomorphism classes of the fiber functors. For $H_{d:-1,1}$, by definition,

$$
\Omega_{g}\left(x^{i} y^{j} z^{k}, x^{l} y^{m} z^{n}\right)=\frac{\omega\left(g x^{i} y^{j} z^{k} g^{-1}, g x^{l} y^{m} z^{n} g^{-1}, g\right) \omega\left(g, x^{i} y^{j} z^{k}, x^{l} y^{m} z^{n}\right)}{\omega\left(g x^{i} y^{j} z^{k} g^{-1}, g, x^{l} y^{m} z^{n}\right)}, \quad \forall g \in G .
$$

Then for any $\pi \in L$,

$$
\Omega_{t \pi}\left(x^{i} y^{j} z^{k}, x^{l} y^{m} z^{n}\right)=\Omega_{t}\left(x^{i} y^{j} z^{k}, x^{l} y^{m} z^{n}\right)=(-1)^{k l+k m+i m} .
$$

Reorganizing the order of $x^{i} y^{j} z^{k}$ to $z^{k} x^{i} y^{j}$, by Proposition 3.4, $\Omega_{t}$ corresponds to the element determined by $(1,1,1)$ in $H^{2}\left(\mathbb{Z}_{2} \times \mathbb{Z}_{2} \times \mathbb{Z}_{2}, \mathbb{k}^{\times}\right)$. Let $[\alpha],[\beta] \in H^{2}\left(\mathbb{Z}_{2} \times \mathbb{Z}_{2} \times \mathbb{Z}_{2}, \mathbb{k}^{\times}\right)$determined by the sequences $\left(a_{12}, a_{13}, a_{23}\right)$ and $\left(b_{12}, b_{13}, b_{23}\right)$, respectively. Observe that $\Omega_{t \pi}=\Omega_{t}$ for all $\pi \in L$. Then by Remark 3.3, fiber functors $(L, \alpha)$ and $(L, \beta)$ are isomorphic, if and only if, the class of $\alpha \beta^{t} \Omega_{t}$ is trivial, if and only if, $\alpha \beta^{t} \Omega_{t}$ is symmetric. By definition of $\alpha, \beta, \beta^{t}$ and $\Omega_{t}$,

$$
\alpha \beta^{t} \Omega_{t}\left(z^{k} x^{i} y^{j}, z^{n} x^{l} y^{m}\right)=(-1)^{\left(a_{12}+b_{13}+1\right) k l+\left(a_{13}+b_{12}+1\right) k m+\left(a_{23}+1\right) i m+b_{23} j l} .
$$


Then $\alpha \beta^{t} \Omega_{t}\left(z^{k} x^{i} y^{j}, z^{n} x^{l} y^{m}\right)$ is symmetric if and only if $b_{23}=0, a_{23}=1, a_{12}+b_{13}+1=a_{13}+b_{12}+1 \equiv 0$ $\bmod 2$. Therefore, elements in $H^{2}\left(\mathbb{Z}_{2} \times \mathbb{Z}_{2} \times \mathbb{Z}_{2}, \mathbb{k}^{\times}\right)$are divided into four classes:

$$
\{(0,0,1),(1,1,0)\}, \quad\{(1,1,1),(0,0,0)\}, \quad\{(1,0,1),(1,0,0)\}, \quad\{(0,1,1),(0,1,0)\} .
$$

Therefore, there are four Galois objects up to isomorphism for $\left(H_{d:-1,1}\right)^{*} \cong H_{d:-1,1}$. For $H_{d: 1,-1}$, the proof follows the same line as for $H_{d:-1,1}$.

For $H_{d:-1,-1}$, we show that the class of $\left.\omega\right|_{L \times L \times L}$ is trivial only when $L=\langle x\rangle \times\langle y\rangle \times\langle z\rangle$ and $\langle x t\rangle \times\langle z\rangle$. If $L=\langle(x t) z, y\rangle$ or $\langle x t, y z\rangle$, then $\omega\left((t x y z)^{i},(t x y z)^{j},(t x y z)^{k}\right)=(-1)^{k\left[\frac{i+j}{2}\right]}$, and hence by Remark 3.6 the class of $\omega$ is not trivial in $H^{3}\left(\mathbb{Z}_{2}, \mathbb{k}^{\times}\right)$when restricted to the subgroup $\left\langle\right.$txyz $\cong \cong \mathbb{Z}_{2}$, which implies that the class of $\left.\omega\right|_{L \times L \times L}$ is not trivial in $H^{3}\left(L, \mathbb{k}^{\times}\right)$. If $L=\langle x\rangle \times\langle y\rangle \times\langle z\rangle$, then completely analogous to the case $H_{d:-1,1}$, there exist four isomorphism classes. If $L=\langle x t\rangle \times\langle z\rangle$, then similar to the proof of Lemma 3.8, the class of $\left.\omega\right|_{L \times L \times L}$ is trivial and direct computations show that $\Omega_{t}$ is not symmetric, which implies that $(L, 1)$ and $(L, \alpha)$ are isomorphic. Consequently, there are five Galois objects for $\left(H_{d:-1,-1}\right)^{*}$.

For $H_{d: 1,1}$, we show that the class of $\left.\omega\right|_{L \times L \times L}$ is trivial when $L=\langle x\rangle \times\langle y\rangle \times\langle z\rangle$ and $\langle x t\rangle \times\langle z\rangle$. Similar to the case $H_{d:-1,-1}$, the fiber functors $(L, \alpha)$ admit five or one isomorphism class if $L=\langle x\rangle \times\langle y\rangle \times\langle z\rangle$ or $\langle x t\rangle \times\langle z\rangle$, respectively. For $L=\langle x t, y z\rangle \cong D_{8}$ or $\langle(x t) z, y\rangle \cong D_{8}$, a direct computation shows that the class of $\left.\Omega_{t}\right|_{L \times L}$ is non-trivial in both cases, then there are at most two Galois objects as in Lemma 3.10. Consequently, the number of Galois objects of $\left(H_{d: 1,1}\right)^{*} \cong H_{d: 1,1}$ ranges from six to eight.

Remark 3.14. From the proof of Lemma 3.13, if $\left.\omega\right|_{\langle x t, y z\rangle \times\langle x t, y z\rangle \times\langle x t, y z\rangle}$ and $\left.\omega\right|_{\langle(x t) z, y\rangle \times\langle(x t) z, y\rangle \times\langle(x t) z, y\rangle}$ are trivial, then $H_{d: 1,1}$ has eight Galois objects; if $\left.\omega\right|_{\langle x t, y z\rangle \times\langle x t, y z\rangle \times\langle x t, y z\rangle}$ and $\left.\omega\right|_{\langle(x t) z, y\rangle \times\langle(x t) z, y\rangle \times\langle(x t) z, y\rangle}$ are not trivial, then $H_{d: 1,1}$ has six Galois objects; otherwise, $H_{d: 1,1}$ has seven Galois objects.

Lemma 3.15. $\left(H_{B: 1}\right)^{*} \cong H_{C: 1}$ and $\left(H_{B: X}\right)^{*} \cong H_{E}$ have only trivial Galois objects up to isomorphism.

Proof. By Lemma 3.1, the possibilities of $L$ are $\langle x\rangle \times\langle y t\rangle \cong \mathbb{Z}_{4} \times \mathbb{Z}_{2},\left\langle x^{2}\right\rangle \times\langle t x\rangle \times\langle y t\rangle \cong \mathbb{Z}_{2} \times \mathbb{Z}_{2} \times \mathbb{Z}_{2}$ $\langle x, t(y t)\rangle=\langle x, y\rangle \cong D_{8},\langle x(y t), t(y t)\rangle=\langle x(y t), y\rangle \cong D_{8}$.

For $H_{B: 1}$, we first claim that $L=\langle x, y\rangle \cong D_{8}$. If $L=\left\langle x^{2}\right\rangle \times\langle t x\rangle \times\langle y t\rangle$ or $\langle x\rangle \times\langle y t\rangle \cong \mathbb{Z}_{4} \times \mathbb{Z}_{2}$, then consider the subgroup $\left\langle t x^{2} y\right\rangle \cong \mathbb{Z}_{2}$ of $L$, we have

$$
\omega\left(\left(t x^{2} y\right)^{i},\left(t x^{2} y\right)^{j},\left(t x^{2} y\right)^{k}\right)=(-1)^{k\left[\frac{i+j}{2}\right]} ;
$$

by Remark 3.6, the class of $\omega$ is not trivial in $H^{3}\left(\mathbb{Z}_{2}, \mathbb{k}^{\times}\right)$when restricted to subgroup $\left\langle t x^{2} y\right\rangle$, which implies that the class of $\left.\omega\right|_{L \times L \times L}$ is non-trivial. If $L=\langle x(y t), y\rangle \cong D_{8}$, then a direct computation shows that restriction of $\omega$ on $\langle x(y t)\rangle \cong \mathbb{Z}_{4}$ is a non-trivial class by Remark 3.6. Clearly, $\left.\omega\right|_{L \times L \times L}=1$ if $L=\langle x, y\rangle \cong D_{8}$. Therefore, the claim follows.

Now we claim that $(L, 1)$ and $(L, \beta)$ are isomorphic, where $[\beta]$ represents $H^{2}\left(D_{8}, \mathrm{k}^{\times}\right)$. Observe that $\Omega_{t}\left(x^{k} y^{l}, x^{m} y^{n}\right)=\xi^{l m}$. If $\Omega_{t}=d(f)$ for some morphism $f: L \rightarrow \mathbb{k}^{\times}$, then $f\left(x^{k}\right)=f(x)^{k}$ and $f(y)^{2}=f\left(y^{2}\right)=f(x)^{4}=1$. Observe that $1=\Omega_{t}\left(x^{3}, y\right)$. Then $f\left(x^{3}\right) f(y)=f\left(x^{3} y\right)=f(x)^{3} f(y)$. Since $\xi^{-1}=\Omega_{t}(y, x)=f(y) f(x) f(y x)^{-1}$, it follows that $-f(x)^{2}=f(y x)^{2}=f\left(x^{3} y\right)^{2}=f(x)^{6} f(y)^{2}=f(x)^{2}$, a contradiction. Therefore, the class of $\left.\Omega_{t}\right|_{L \times L}$ is not trivial and then the claim follows by Remark 3.7. Consequently, there are only trivial Galois objects for $\left(H_{B: 1}\right)^{*} \cong H_{C: 1}$.

For $H_{B: X}$, the proof follows the same line as for $H_{B: 1}$.

Lemma 3.16. Up to isomorphism $H_{B: 1} \cong\left(H_{C: 1}\right)^{*}$ has three Galois objects, and $H_{C: \sigma_{1}}$ has only trivial Galois objects.

Proof. By Lemma 3.1, the possibilities of $L$ are $\langle t y\rangle \cong \mathbb{Z}_{8}$ and $\langle x, y\rangle \cong D_{8}$.

For $\left(H_{C: \sigma_{1}}\right)^{*} \cong H_{C: \sigma_{1}}$, we first claim that $L \neq\langle t y\rangle \cong \mathbb{Z}_{8}$. Indeed, if the class of $\left.\omega\right|_{L \times L \times L}$ is trivial, then by Remark 3.6, there must be a 2-cochain $\varphi: L \times L \rightarrow \mathbb{k}^{\times}$such that $\omega d^{2}(\varphi)=\omega_{a}$ for some 
$0 \leq a<8$. Then $\omega\left((t y),(t y)^{j},(t y)\right)=\nu_{8}^{a}$ if $j=7$ otherwise it is equal to 1 . In particular, $\nu_{8}^{a}=$ $\mu^{-1} \frac{\varphi\left((t y)^{7},(t y)\right)}{\varphi\left((t y),(t y)^{7}\right)}$, where $\mu$ is a primitive 8-th root of unity, and induction on $j$ shows that $\nu_{8}^{a}=\mu^{2} \neq 1$.

Assume that $L=\langle x, y\rangle \cong D_{8}$. Clearly, $\left.\omega\right|_{L \times L \times L}=1$. Now we determine the isomorphism classes of fiber functors. By definition, $\Omega_{t}\left(x^{k} y^{l}, x^{m} y^{n}\right)=\xi^{l(n-m)}$. We claim that the class of $\Omega_{t}$ is not trivial in $H^{2}\left(D_{8}, \mathbb{k}^{\times}\right)$. Indeed, if $\Omega_{t}=d(f)$ for some morphism $f: D_{8} \rightarrow \mathbb{k}^{\times}$, then from $\Omega_{t}(y, y)=1=\Omega_{t}(x, y)$, we have $f(y)^{2}=1$ and $f(x y)=f(x) f(y)$; since $\Omega_{t}(x y, y)=\xi$, it follows that $\xi f(x)=f(x y) f(y)$, $-f(x)^{2}=f(x y)^{2} f(y)^{2}=f(x)^{2}$, a contradiction. Consequently, there is only one Galois object up to isomorphism for $H_{C: \sigma_{1}}$ by Remark 3.7 .

For $H_{C: 1}, \omega(\tau, \sigma)$ is trivial, so is $\Omega_{g}$ for $\forall g \in G$. Hence by Theorem 2.2, Lemma 3.1 and Proposition 3.2, there are three Galois objects for $\left(H_{C: 1}\right)^{*} \cong H_{B: 1}$.

Lemma 3.17. There are two Galois objects up to isomorphism for $\left(H_{E}\right)^{*} \cong H_{B: X}$.

Proof. In this case, $\omega(\tau, \sigma)$ is trivial. Then by Lemma 3.1, $L=\langle t y\rangle \cong \mathbb{Z}_{8}$ or $\langle x, y\rangle \cong Q_{8}$. Consequently, the lemma follows by Proposition 3.2 and Theorem 2.2 .

In summary, we have the following theorem:

Theorem 3.18. Let $H$ be a semisimple Hopf algebra of dimension 16 in [Ka, Table 1], up to isomorphism the number of Galois objects of $H^{*}$ is given as follows:

(1) $\left(H_{C: \sigma_{1}}\right)^{*} \cong H_{C: \sigma_{1}},\left(H_{c: \sigma_{1}}\right)^{*},\left(H_{B: 1}\right)^{*} \cong H_{C: 1}$ and $\left(H_{B: X}\right)^{*} \cong H_{E}$ only have one trivial Galois object.

(2) $\left(H_{E}\right)^{*} \cong H_{B: X}$ and $\left(H_{c: \sigma_{0}}\right)^{*}$ have two Galois objects.

(3) $\left(H_{C: 1}\right)^{*} \cong H_{B: 1}$ and $\left(H_{a: y}\right)^{*}$ have three Galois objects.

(4) $\left(H_{d:-1,1}\right)^{*} \cong H_{d:-1,1}$ has four Galois objects.

(5) $\left(H_{d:-1,-1}\right)^{*} \cong H_{c: \sigma_{1}}$ and $\left(H_{d: 1,-1}\right)^{*} \cong H_{b: 1}$ have five Galois objects.

(6) $\left(H_{b: x^{2} y}\right)^{*}$ and $\left(H_{b: y}\right)^{*}$ have seven Galois objects.

(7) $\left(H_{a: 1}\right)^{*}$ and $\left(H_{b: 1}\right)^{*}$ have one or two Galois objects, the number of Galois objects of $\left(H_{d: 1,1}\right)^{*} \cong$ $H_{d: 1,1}$ ranges from six to eight.

Remark 3.19. Masuoka has already proved that $\mathcal{B}_{16} \cong H_{C: \sigma_{1}}$ has only trivial Galois objects and $\mathcal{A}_{16} \cong H_{B: 1}$ has three Galois objects up to isomorphism, see [Ma2, Theorem 4.8] for details.

\section{Cocycle Deformations of Semisimple Hopf algebras of Dimension 16}

In this section, we determine cocycle deformations of semisimple Hopf algebras of dimension 16 listed in [Ka, Table 1] using their Frobenius-Schur indicators and Grothendieck rings. The Grothendieck rings of these Hopf algebras have been determined by Kashina (see [Ka, Table 1]) and the second indicators of irreducible representations have been computed in [KMM].

We first determine twist equivalence classes of these Hopf algebras.

Lemma 4.1. The Hopf algebras $H_{a: 1}, H_{a: y}, H_{b: y}, H_{b: x^{2} y},\left(H_{c: \sigma_{1}}\right)^{*} \cong H_{d:-1,-1}$ and $\left(H_{b: 1}\right)^{*} \cong H_{d: 1,-1}$ have different Frobenius-Schur indicator sets and hence they are pairwise Drinfeld twist inequivalent.

Proof. Clearly, it is not enough to distinguish all the Drinfeld twist equivalence classes via the second indicators. We shall prove the lemma by computing higher Frobenius-Schur indicators. A direct computation shows that the third indicators are not sufficient to distinguish them. Here we omit the computation details, because the expression of the Sweedler power of the integral is too long. Let $\chi_{1}$, $\chi_{2}$ be the characters of 2-dimensional irreducible representations of these Hopf algebras, respectively. 
The fourth Sweedler power of integral $\Lambda$ with $\varepsilon(\Lambda)=1$ is given by

$$
\Lambda^{[4]}= \begin{cases}\frac{3+y}{4}, & H \cong H_{a: 1} \text { or } H_{a: y} \\ \frac{3}{2}, & H \cong H_{b: y} \\ 1, & H \cong H_{d: 1,-1} \\ \frac{1+x y}{2}, & H \cong H_{d:-1,-1}\end{cases}
$$

For $H_{a: 1}$, the indicators are: $\nu_{2}\left(\chi_{1}\right)=-1, \nu_{2}\left(\chi_{2}\right)=1 ; \nu_{3}\left(\chi_{1}\right)=\nu_{3}\left(\chi_{2}\right)=0 ; \nu_{4}\left(\chi_{1}\right)=\nu_{4}\left(\chi_{2}\right)=1$.

For $H_{a: y}$, the indicators are: $\nu_{2}\left(\chi_{1}\right)=\nu_{2}\left(\chi_{2}\right)=1 ; \nu_{3}\left(\chi_{1}\right)=\nu_{3}\left(\chi_{2}\right)=0 ; \nu_{4}\left(\chi_{1}\right)=\nu_{4}\left(\chi_{2}\right)=1$.

For $H_{b: y}$, the indicators are: $\nu_{2}\left(\chi_{1}\right)=1, \nu_{2}\left(\chi_{2}\right)=-1 ; \nu_{3}\left(\chi_{1}\right)=\nu_{3}\left(\chi_{2}\right)=0 ; \nu_{4}\left(\chi_{1}\right)=\nu_{4}\left(\chi_{2}\right)=3$.

For $H_{b: x^{2} y}$, the indicators are: $\nu_{2}\left(\chi_{1}\right)=\nu_{2}\left(\chi_{2}\right)=-1$.

For $H_{d:-1,-1}$, the indicators are: $\nu_{2}\left(\chi_{1}\right)=\nu_{2}\left(\chi_{2}\right)=1 ; \nu_{3}\left(\chi_{1}\right)=\nu_{3}\left(\chi_{2}\right)=\nu_{4}\left(\chi_{1}\right)=\nu_{4}\left(\chi_{2}\right)=0$.

For $H_{d: 1,-1}$ the indicators are: $\nu_{2}\left(\chi_{1}\right)=\nu_{2}\left(\chi_{2}\right)=1, \nu_{3}\left(\chi_{1}\right)=\nu_{3}\left(\chi_{2}\right)=0, \nu_{4}\left(\chi_{1}\right)=\nu_{4}\left(\chi_{2}\right)=2$.

Consequently, these Hopf algebras have different Frobenius-Schur indictor sets. Then by Theorem 2.15, they are pairwise Drinfeld twist inequivalent.

Theorem 4.2. Let $H$ and $K$ be two non-isomorphic semisimple Hopf algebras of dimension 16 listed in [Ka, Table 1]. Then $H$ and $K$ are twist inequivalent.

Proof. From the second Frobenius-Schur indicators of the 2-dimensional irreducible representations [KMM], we obtain six possible twist equivalence classes:

$$
\begin{aligned}
& \left\{H_{a: 1}, H_{b: 1}, H_{B: X}\right\},\left\{H_{b: x^{2} y}\right\},\left\{H_{c: \sigma_{0}}, H_{c: \sigma_{1}}\right\},\left\{H_{C: 1}, H_{C: \sigma_{1}}\right\}, \\
& \left\{H_{E}\right\},\left\{H_{b: y}, H_{a: y}, H_{B: 1}, H_{d: 1,1}, H_{d:-1,1}, H_{d: 1,-1}, H_{d:-1,-1}\right\} .
\end{aligned}
$$

Recall that the Grothendieck ring is a gauge invariant. From the Grothendieck rings of semisimple Hopf algebras listed in [Ka, Table 1], the possible classes $\left\{H_{c: \sigma_{0}}, H_{c: \sigma_{1}}\right\},\left\{H_{a: 1}, H_{b: 1}, H_{B: X}\right\}$ and $\left\{H_{C: 1}, H_{C: \sigma_{1}}\right\}$ are pairwise twist inequivalent, and the last possible class is divided into three possible equivalence classes:

$$
\left\{H_{B: 1}\right\},\left\{H_{b: y}, H_{a: y},\left(H_{b: 1}\right)^{*},\left(H_{c: \sigma_{1}}\right)^{*}\right\},\left\{H_{d:-1,1}, H_{d: 1,1}\right\} .
$$

The Hopf algebras in the second possible class are pairwise twist inequivalent by Theorem 2.15 and Lemma 4.1. Observe that $H_{d: 1,1}=\mathbb{k}\left[D_{8} \times \mathbb{Z}_{2}\right]^{J}, H_{d:-1,1}=H_{8} \otimes \mathbb{k}\left[\mathbb{Z}_{2}\right], H_{d: 1,-1} \cong\left(H_{b: 1}\right)^{*}, H_{d:-1,-1} \cong$ $\left(H_{c: \sigma_{1}}\right)^{*}$, and $\mathbb{k}\left[D_{8} \times \mathbb{Z}_{2}\right]^{J}$ is triangular while $H_{8} \otimes \mathbb{k}\left[\mathbb{Z}_{2}\right]$ is not triangular. Then the Hopf algebras in the third class are pairwise twist inequivalent.

Next we determine whether these Hopf algebras in [Ka, Table 1] are Drinfeld twists of group algebras. By Theorem 2.5, semisimple Hopf algebras with the same Grothendieck ring are differed by a 2-pseudococycle. Therefore, it suffices to distinguish a 2-pseudo-cocycle is a Drinfeld twist or not.

Lemma 4.3. The Hopf algebras $H_{a: 1}, H_{a: y}, H_{b: y}, H_{b: x^{2} y},\left(H_{c: \sigma_{1}}\right)^{*}$ and $\left(H_{b: 1}\right)^{*}$ with the same Grothendieck ring $\operatorname{Gr}\left(G_{5}\right) \cong \operatorname{Gr}\left(G_{6}\right)$ are not twist deformations of the group algebra $\mathbb{k}\left[G_{5}\right]$ or $\mathbb{k}\left[G_{6}\right]$.

Proof. We prove the statements by showing that their Frobenius-Schur indicator sets are different from those of $G_{5}$ and $G_{6}$. For $G_{5}:=\left\langle a, b \mid a^{4}=b^{4}=1, b a=a^{-1} b\right\rangle$, there are two self-dual 2-dimensional irreducible representations $\pi_{1}, \pi_{2}$ with characters $\chi_{1}, \chi_{2}$ given by

$$
\pi_{1}(a)=\left(\begin{array}{cc}
0 & 1 \\
1 & 0
\end{array}\right), \pi_{1}(b)=\left(\begin{array}{cc}
\theta & 0 \\
0 & -\theta
\end{array}\right) ; \pi_{2}(a)=\left(\begin{array}{cc}
0 & \theta \\
\theta & 0
\end{array}\right), \pi_{2}(b)=\left(\begin{array}{cc}
\theta & 0 \\
0 & -\theta
\end{array}\right)
$$

where $\theta$ is a 4 -th root of unity and the integral element $\Lambda=\frac{1}{16} \Sigma_{i, j=0}^{3} a^{i} b^{j}$. Then a direct computation shows that the higher Frobenius-Schur indicators are: $\nu_{2}\left(\chi_{1}\right)=1, \nu_{2}\left(\chi_{2}\right)=-1 ; \nu_{3}\left(\chi_{1}\right)=\frac{-\theta}{4}, \nu_{3}\left(\chi_{2}\right)=$ $0 ; \nu_{4}\left(\chi_{1}\right)=\nu_{4}\left(\chi_{2}\right)=2$. 
For $G_{6}:=\left\langle a, b, c \mid a^{4}=b^{2}=c^{2}=1, b a b=a c\right\rangle$, there are two self-dual 2-dimensional irreducible representations $\pi_{1}, \pi_{2}$ with characters $\chi_{1}, \chi_{2}$ given by

$$
\begin{aligned}
& \pi_{1}(a)=\left(\begin{array}{cc}
0 & -1 \\
1 & 0
\end{array}\right), \quad \pi_{1}(b)=\left(\begin{array}{cc}
0 & 1 \\
1 & 0
\end{array}\right), \quad \pi_{1}(c)=\left(\begin{array}{cc}
-1 & 0 \\
0 & -1
\end{array}\right) ; \\
& \pi_{2}(a)=\left(\begin{array}{cc}
0 & -\theta \\
\theta & 0
\end{array}\right), \quad \pi_{2}(b)=\left(\begin{array}{cc}
0 & 1 \\
1 & 0
\end{array}\right), \quad \pi_{2}(c)=\left(\begin{array}{cc}
-1 & 0 \\
0 & -1
\end{array}\right)
\end{aligned}
$$

and the integral element $\Lambda=\frac{1}{16} \sum_{\substack{0 \leq i \leq 3, 0 \leq j, k \leq 1}} a^{i} b^{j} c^{k}$. Then a direct computation shows that higher FrobeniusSchur indicators of $\mathbb{k}\left[G_{6}\right]$ are: $\nu_{2}\left(\chi_{1}\right)=\nu_{2}\left(\chi_{2}\right)=1 ; \nu_{3}\left(\chi_{1}\right)=\nu_{3}\left(\chi_{2}\right)=0 ; \nu_{4}\left(\chi_{1}\right)=\nu_{4}\left(\chi_{2}\right)=2$.

By Theorem 2.15 and Lemma 4.1, the proof is completed.

Theorem 4.4. Except the Hopf algebras $H_{E} \cong \mathbb{k}\left[G_{2}\right]^{J}, H_{C: 1} \cong \mathbb{k}\left[D_{16}\right]^{J}$ and $H_{d: 1,1} \cong \mathbb{k}\left[D_{8} \times \mathbb{Z}_{2}\right]^{J}$, the semisimple Hopf algebras of dimension 16 in [Ka, Table 1] are not twist equivalent to group algebras.

Proof. From [Ka, Table 1], there are 7 isomorphism classes of Grothendieck rings, that is, $\operatorname{Gr}\left(G_{1}\right)$, $\operatorname{Gr}\left(G_{2}\right), G r\left(G_{5}\right), G r\left(G_{7}\right), G r\left(D_{16}\right), G r\left(D_{8} \times \mathbb{Z}_{2}\right)$ and the only non-commutative fusion ring $K_{5,5}$. Moreover, $H_{E} \cong \mathbb{k}\left[G_{2}\right]^{J}, H_{C: 1} \cong \mathbb{k}\left[D_{16}\right]^{J}$ and $H_{d: 1,1} \cong \mathbb{k}\left[D_{8} \times \mathbb{Z}_{2}\right]^{J}$.

Now we claim that the Hopf algebras $H$ in [Ka, Table 1] are not twist deformations of group algebras except for $H_{E}, H_{C: 1}$ and $H_{d: 1,1}$. By Theorem 2.5, it suffices to show $H$ is not twist equivalent to $\mathbb{k}\left[G_{i}\right]$ when $\operatorname{Gr}(H) \cong G r\left(G_{i}\right)$.

If $H \cong H_{d:-1,1}$, then $H_{d:-1,1} \cong H_{8} \otimes \mathbb{k}\left[\mathbb{Z}_{2}\right]$ and $G r(H) \cong G r\left(D_{8} \times \mathbb{Z}_{2}\right) \cong G r\left(Q_{8} \times \mathbb{Z}_{2}\right)$. This case has been stated in Theorem 4.2. If $H \cong H_{C, \sigma_{1}}$, then $\operatorname{Gr}\left(H_{C, \sigma_{1}}\right) \cong G r\left(D_{16}\right)=G r\left(Q_{16}\right)$. Since $H_{C: 1}$ is the only Drinfeld twist deformation of $\mathbb{k}\left[D_{16}\right]$, and $\mathbb{k}\left[Q_{16}\right]$ does not have any non-trivial twist deformation [Ma2, it follows that $H_{C: \sigma_{1}}$ is not Drinfeld twist equivalent to $\mathbb{k}\left[D_{16}\right]$ or $\mathbb{k}\left[Q_{16}\right]$. By Theorem 3.18 , the dual Hopf algebras of $H_{c, \sigma_{1}}, H_{B, 1}$ and $H_{B, X}$ have only trivial Galois objects. Therefore, they are not twist equivalent to group algebras. For $H_{a, 1}, H_{b, 1}$ and $H_{c, \sigma_{0}}$, their Frobenius-Schur indicator sets are different from group algebras by Lemma 4.3 and $[\mathrm{KMM}]$. Consequently, the proof is completed by Theorem 2.15.

Remark 4.5. Note that $H_{d:-1,1} \cong H_{8} \otimes \mathbb{k}\left[\mathbb{Z}_{2}\right]$. It turns out in [NS1] that $H_{8}, \mathbb{k}\left[Q_{8}\right]$ and $\mathbb{k}\left[D_{8}\right]$ have different Frobenius-Schur indicator sets; if $H \cong H_{B: 1}$ or $H_{B: X}$, then $G r(H) \cong K_{5,5}$, which is non-commutative [Ka]. Then the statements for $H_{d:-1,1} H_{B: 1}$ and $H_{B: X}$ in Theorem 4.4 follow.

Finally, we determine cocycle deformations of semisimple Hopf algebras listed in [Ka, Table 1].

Lemma 4.6. The Hopf algebras $H_{B: 1}, H_{B: X}, H_{d: 1,1}$ listed in [Ka, Table 1] have only one non-trivial cocycle deformation.

Proof. Observe that $H_{E}=\mathbb{k}\left[G_{2}\right]^{J_{1}}, H_{C: 1}=\mathbb{k}\left[D_{16}\right]^{J_{2}}, H_{B: X} \cong H_{E}^{*}$ and $H_{B: 1} \cong\left(H_{C: 1}\right)^{*}$. Then $H_{B: X}=\left(\mathbb{k}^{G_{2}}\right)^{\sigma}$ and $H_{B: 1}=\left(\mathbb{k}^{D_{16}}\right)^{\mu}$ for some non-trivial 2-cocycles $\sigma, \mu$. By Theorem 3.18, $H_{B: X}$ has two Galois objects. Hence $H_{B: X}$ has only one non-trivial 2-cocycle deformation. Since $\mathbb{k}\left[D_{16}\right]$ has only one non-trivial Drinfeld twist Ma2, it follows that $H_{B: 1}$ has only one non-trivial cocycle deformation.

Since $H_{d: 1,1}$ is self-dual and isomorphic to $\mathrm{k}\left[D_{8} \times \mathbb{Z}_{2}\right]^{J}$ for some Drinfeld twist $J$, it follows that $H_{d: 1,1} \cong\left(\mathbb{k}^{D_{8} \times \mathbb{Z}_{2}}\right)^{\sigma}$ for some non-trivial 2-cocycle $\sigma$. By Theorem 4.4, among the three Hopf algebras with Grothendieck ring $G r\left(\left[D_{8} \times \mathbb{Z}_{2}\right]\right), H_{d: 1,1}$ is the only twist deformation of $\mathbb{k}\left[D_{8} \times \mathbb{Z}_{2}\right]$, which implies that $H_{d: 1,1}$ has only one non-trivial 2-cocycle deformation.

Theorem 4.7. The Hopf algebras $H_{B: 1}, H_{B: X}, H_{d: 1,1}$ have only one non-trivial cocycle deformation, and others in [Ka, Table 1] have only trivial cocycle deformations. 
Proof. Let $H$ be a semisimple Hopf algebra listed in [Ka, Table 1]. If $H$ admits a non-trivial cocycle deformation $\sigma$, then $\left(H^{\sigma}\right)^{*} \cong\left(H^{*}\right)^{J}$, that is, $H^{*}$ admits a non-trivial Drinfeld twist $J$. By Theorems 4.2 and $4.4,\left(H^{*}\right)^{J}$ is isomorphic to $\mathbb{k}\left[G_{2}\right], \mathbb{k}\left[D_{16}\right]$, or $\mathbb{k}\left[D_{8} \times \mathbb{Z}_{2}\right]$, equivalently $H$ is a cocycle deformation of a dual group algebra. Then the theorem follows by Theorems $4.2 \& 4.4$ and Lemma 4.6 ,

\section{ACKNOWLEDGEMENTS}

The authors would like to appreciate the continued encouragement and help from their PhD supervisor Prof. Naihong Hu. This article started after reading [CMNVW], we are grateful to the authors in [CMNVW], specially thanks to Prof. Chelsea Walton for her valuable suggestions and comments on earlier version of the manuscript. The authors thank the referees for pointing errors out and their comments on improving this paper.

\section{REFERENCES}

[BPW] G. Benkart, M. Pereira and S. Witherspoon, Yetter-Drinfeld modules under cocycle twists, J. Algebra. 324 (2010), 2990-3006.

[CMNVW] A. M. Castano, S. Montgomery, S. Natale, M. D. Vega and C. Walton, Cocycle deformations and Galois objects for semisimple Hopf algebras of dimension $p^{3}$ and $p q^{2}$, J. Pure Appl. Algebra. 222 (7) (2018), 1643-1669.

[EGNO] P. Etingof, S. Gelaki, D. Nikshych and V. Ostrik, Tensor categories, AMS Mathematical Surveys and Monographs 205 (2015).

[ENO1] P. Etingof, D. Nikshych and V. Ostrik, On fusion categories, Ann. of Math. 162 (2) (2005), 581-642.

[ENO2] - Weakly group-theoretical and solvable fusion categories, Adv. Math. 226 (2011), 176-205.

[EV] M. Enock and L. Vainerman, Deformation of the Kac algebra by an abelian subgroup, Comm. Math. Phys $\mathbf{1 7 8}$ (1996), 571-596.

[H] I. Hofstetter, Extensions of Hopf algebras and their cohomological description, J. Algebra 164 (1994), $264-298$.

[HLYY] H. Huang, G. Liu, Y, Yang and Y. Ye, Finite quasi-quantum groups of diagonal type, J. Reine Angew. Math, to appear, arXiv:1611.04096

[K] G. I. Kac, Extensions of groups to ring groups, Math. USSR Sb. 5 (1968), 451-474.

[Kar] G. Karpilovsky, The Schur multiplier, (1987), Oxford University press.

[Ka] Y. Kashina, Classification of semisimple Hopf algebras of dimension 16, J. Algebra 232 (2000), 617-663.

[KMM] Y. Kashina, G. Mason, and S. Montgomery, Computing the Frobenius-Schur indicator for abelian extensions of Hopf algebras, J. Algebra 251 (2002), 888-913.

[KSZ] Y. Kashina, Y. Sommerhäuer and Y. Zhu, On higher Frobenius-Schur indicators, Mem. Amer. Math. Soc. 181 (2006).

[LM] V. Linchenko and S. Montgomery, A Frobenius-Schur theorem for Hopf algebras, Algebra. Represent. Theory 3 (2000), 347-355.

[Ma1] A. Masuoka, Self-dual Hopf algebras of dimension p $p^{3}$ obtained by extension, J. Algebra 178 (1995), 791-806.

[Ma2] - Cocycle deformations and Galois objects for some cosemisimple Hopf algebras of finite dimension, Contem. Math 267 (2000).

[Ma3] —, Hopf algebra extensions and cohomology, Math. Sci. Res. Inst. Publ. 43 (2002), 167-209.

[MN] G. Mason and S. H. Ng, Central invariants and Frobenius-Schur indicators for semisimple quasi-Hopf algebras, Adv. Math 190 (2005), 161-195.

[Na1] S. Natale, On semisimple Hopf algebras of dimension $p q^{2}$, J. Algebra 221 (1) (1999), 242-278.

[Na2] -, On semisimple Hopf algebras of dimension $p q^{2}$. II, Algebr. Reprsent. Theory 4 (3) (2001), $277-291$.

[Na3] -, On group theoretical Hopf algebras and exact factorizations of finite groups, J. Algebra 270 (2003), $199-211$.

[Na4] —, On the equivalence of module categories over a group-theoretical fusion category, SIGMA 13 (042) (2017), 9 pp.

[Ni] D. Nikshych, Ko-rings and twisting of finite-dimensional semisimple Hopf algebras, Comm. Algebra 26 (1) (1998), 321-342; Erratum: $K_{0}$-rings and twisting of finite-dimensional semisimple Hopf algebras, Comm. Algebra 26 (4) (1998), 1347.

[NS1] S-H. Ng and P. Schauenburg, Central invariants and higher indicators for semisimple quasi-Hopf algebras, Trans. Amer. Math. Soc. 360 (4) (2008), 1839-1860.

[NS2] —, Frobenius-Schur indicators and exponents of spherical categories, Adv. Math 211 (2007), 34-71.

[O1] V. Ostrik, Module categories, weak Hopf algebras and modular invariants, Transform. Groups 8 (2003), $177-206$.

[O2] -, Module categories over the Drinfeld double of a finite group, Int. Math. Res. Not. 2003 (27) (2003), 1507-1520. 
[S1] P. Schauenburg, Hopf bi-Galois extensions, Comm. Algebra 24 (1996), 3797-3825.

[Ul] K. H. Ulbrich, Galois extensions as functors of comodules, Manuscr. Math. 59 (1987), 391-397.

Rongchuan Xiong: School of Mathematical Sciences, East China Normal University, Shanghai 200241, CHINA

E-mail address: rcxiong@foxmail.com

Zhiqiang Yu: School of Mathematical Sciences, East China Normal University, Shanghai 200241, China

E-mail address: zhiqyu-math@hotmail.com 